\title{
KP-KdV Hierarchy and Pseudo-Differential Operators
}

\author{
Ahmed Lesfari ${ }^{1 *}$
}

\begin{abstract}
The study of KP-KdV equations are the archetype of integrable systems and are one of the most fundamental equations of soliton phenomena and a topic of active mathematical research. Our purpose here is to give a motivated and a sketchy overview of this interesting subject. One of the objectives of this paper is to study the $\mathrm{KdV}$ equation and the inverse scattering method (based on Schrödinger and Gelfand-Levitan equations) used to solve it exactly. We study some generalities on the algebra of infinite order differential operators. The algebras of Virasoro and Heisenberg, nonlinear evolution equations such as the KdV, Boussinesq and KP play a crucial role in this study. We make a careful study of some connection between pseudo-differential operators, symplectic structures, KP hierarchy and tau functions based on the Sato-Date-Jimbo-Miwa-Kashiwara theory. A few other connections and ideas concerning the KdV and Boussinesq equations, the Gelfand-Dickey flows, the Heisenberg and Virasoro algebras are given.
\end{abstract}

Keywords: Gelfand-Levitan integral equation, Integrable systems, KdV equation, KP hierarchy, Schrödinger equation, Symplectic structures.

2010 AMS: Primary 70H06, Secondary 14H55, 14H70, 14K20

${ }^{1}$ Department of Mathematics, Faculty of Sciences, University of Chouaïb Doukkali, B.P. 20, 24000 El Jadida, Morocco, ORCID

orcid.org/0000-0001-6213-4301

*Corresponding author: lesfariahmed@yahoo.fr

Received: 5 November 2018, Accepted: 8 March 2019, Available online: 27 June 2019

\section{Introduction}

Korteweg and de Vries have established a nonlinear partial differential equation describing the gravitational wave propagating in a shallow channel [1] and possessing remarkable mathematical properties :

$$
\frac{\partial u}{\partial t}-6 u \frac{\partial u}{\partial x}+\frac{\partial^{3} u}{\partial x^{3}}=0
$$

where $u(x, t)$ is the amplitude of the wave at the point $x$ and the time $t$. The equation thus bearing their name (abbreviated KdV) admits a solution: the soliton or solitary wave. In fact, this model was obtained from Euler's equations (assuming irrotational flow) by Boussinesq around 1877 (see [2], p. 360) and rediscovered by Korteweg and de Vries in 1890. The solution to this equation was obtained and interpreted rigorously only in the early 1970s while a solitary wave was already observed in 1834 by engineer Scott Russell riding on the Edinburgh Glasgow Canal in Scotland; he described his observation of a hydrodynamic phenomenon as follows : "I was observing the motion of a boat which was rapidly drawn along a narrow channel by a pair of horses, when the boat suddenly stopped - not so the mass of watering the channel which it had put in motion; it accumulated round the prow of the vessel in a state of violent agitation, then suddenly leaving it behind, rolled forward with great velocity, assuming the form of a large solitary elevation, a rounded, smooth and well-defined heap of water, which continued its course along the channel apparently without change of form or diminution of speed. I followed it on horseback, and overtook it still 
rolling on at a rate of some eight or nine miles an hour, preserving its original figure some thirty feet long and a foot to a foot and a half in height. Its height gradually diminished, and after a chase of one or two miles I lost it in the windings of the channel. Such, in the month of August 1834, was my first chance interview with that singular and beautiful phenomenon which I have called the wave of translation ". Fascinated by this phenomenon, Scott-Russell built a wave pool in his garden and worked to generate and study these waves more carefully. This led to a paper [3] dubbed "The report on waves" published in 1844 by the British Association for the Advancement of Science.

A little later, Boussinesq, then Korteweg and de Vries proposed equation (1.1) to explain this phenomenon. The KdV equation preserves mass, momentum, energy, and many other quantities. Many experiments have uncovered the astonishing properties of the solutions of this equation satisfying zero boundary conditions : when $|t| \longrightarrow \infty$, these solutions are decomposed into solitons, i.e., in waves of defined forms progressing at different speeds. These waves propagate over long distances without deformation and one of the remarkable characteristics of solitons is that they are exceptionally stable with respect to disturbances; the term $u \frac{\partial u}{\partial x}$ leads to shock waves while the term $\frac{\partial^{3} u}{\partial x^{3}}$ produces a scattering effect. Everyone can contemplate solitons where the tide comes to die on the beaches. In the field of hydrodynamics for example, tsunamis (tidal waves) are manifestations of solitons. Generally, we group together under the term soliton solutions of nonlinear wave equations presenting the following characteristic properties : they are localized in space, last indefinitely and retain their amplitude and velocity even at the end of several collisions with other solitons. Solitons have become indispensable for the study of several phenomena. In particular, the study of wave propagation in hydrodynamics, the study of localized waves in astrophysical plasmas, They are involved in the study of signals in optical fibers, charge transport phenomena in conductive polymers, localized modes in magnetic crystals, etc. Industrialized societies have developed, after soliton studies, what may be called solitary lasers. The latter play an important role in the field of telecommunications. Ultra-short light signals sent in certain optical fibers made from a specific material can travel long distances without lengthening or fading. The construction of memories with ultra-fast communication time and low energy consumption, is based on the movement of magnetic vortices in the dielectric junction between two superconductors. At the molecular level, the theory of solitons can elucidate the contraction mechanism of striated muscles, the dynamics of biological macromolecules such as DNA and proteins. In the peptide and hydrogen chain of proteins, solitons arise from the marriage of dispersion due to intrapeptide vibrations and the non-linearity due to the interaction of these vibrations with the displacements of peptide groups around their position balanced. But also the theory of solitons had an impact on pure mathematics; for example, it provides the answer to the famous Schottky problem, posited a century ago, on the relations between the periods coming from a Riemann surface. Roughly, it is a question of finding criteria so that a matrix of the periods belonging to the Siegel half-space is the matrix of the periods of a Riemann surface. Geometrically, Schottky's problem consists in characterizing the Jacobians among all the Abelian mainly polarized varieties. In addition to the KdV equation, examples that may be mentioned among the nonlinear equations having soliton-type solutions are: the non-linear equation of Kadomtsev-Petviashvili, the nonlinear Schrödinger equation, the Sine Gordon equation, the Boussinesq equation, the Camassa-Holm equation, the Toda lattice consisting of vibrating masses arranged on a circle and interconnected by springs whose return force is exponential, the non-linear Klein Gordon equation, the Zabusky-Kruskal equation for the Fermi-Pasta-Ulam model of phonons in anharmonic lattice, and so on.

\section{Stationary Schrödinger equation and integral Gelfand-Levitan equation}

Since the method (discussed later) of solving the $\mathrm{KdV}$ equation is based on the idea of studying it in the form of an equation of a certain operator and using the analogy with quantum mechanic, we will expose certain mathematical notions of this mechanic. The terminology of the physicists will be used to describe the properties of the solutions of the stationary Schrödinger's equation,

$$
\frac{\hbar}{2 m} \psi^{\prime \prime}+(\lambda-u(x)) \psi=0, \quad{ }^{\prime} \equiv \frac{d}{d x},
$$

without stopping on the physical motivations of the introduced notions. We will see that the method of the inverse diffusion is reduced to the solution of a linear integral equation (Gelfand-Levitan equation). In the following, we will simplify the notation by using a system of units in which the Planck constant is $\hbar=1$ and the mass of the particle is $m=\frac{1}{2}$. So consider the equation

$$
\psi^{\prime \prime}+(\lambda-u(x)) \psi=0, \quad-\infty<x<\infty
$$

where $\psi$ (unknown) is the wave function of the particle, the spectral parameter $\lambda$ is the energy of the particle, the function $u(x)$ is the potential or potential energy of the particle. This potential is assumed to have a compact support, i.e., is different from zero only in some domain. When the particle is free (i.e., $u=0)$ and has a positive energy (i.e., $\lambda=k^{2}$ ), then equation $(2.1)$ is 
reduced to

$$
\psi^{\prime \prime}(x)+k^{2} \psi=0
$$

and admits two linearly independent solutions $e^{i k x}$ (describing the particle moving to the right) and $e^{-i k x}$ (describing the particle moving to the left).

Let us denote by $E_{(2)}^{s r}\left(\right.$ resp. $\left.E_{(2)}^{s c}\right)$ the space of real (or complex) solutions of equation (2.1) and by $E_{(3)}^{s r}\left(\right.$ resp. $\left.E_{(3)}^{s c}\right)$ the space of the real (or complex) solutions of equation (2.2). The space $E_{(3)}^{s r}\left(\operatorname{resp} . E_{(3)}^{s c}\right)$ is provided with the base $(\cos k x, \sin k x)$ (resp. $\left(e^{i k x}, e^{-i k x}\right)$ ). Let $[\alpha, \beta]$ be the bounded support of $u$. The monodromy operator of equation (2.1) is a linear operator defined by

$$
\mathscr{M}: E_{(2)}^{s r} \longrightarrow E_{(2)}^{s r}, \quad a \cos k x+b \sin k x \longmapsto \begin{cases}a \cos k x+b \sin k x & \text { si } x<\alpha \\ c \cos k x+d \sin k x & \text { si } x>\beta,\end{cases}
$$

where $a, b$ are constants and $(c, d)=\mathscr{M}_{u}(a, b)$. This means that for each solution of equation (2.2) is associated : $(i)$ the solution of (2.1) which is to the left of $\alpha$; in this region the solution of (2.2) coincides with that of (2.1). (ii) the solution of (2.1) which is to the right of $\beta$. Similarly, the complex monodromy operator of equation (2.1) is defined by

$$
\mathscr{M}: E_{(2)}^{s c} \longrightarrow E_{(2)}^{s c}, \quad a e^{i k x}+b e^{-i k x} \longmapsto \begin{cases}a e^{i k x}+b e^{-i k x} & \text { si } x<\alpha \\ c e^{i k x}+d e^{-i k x} & \text { si } x>\beta\end{cases}
$$

Recall that a particle propagating from $x=-\infty$, crosses a potential barrier with a transmission coefficient $T$ and a reflection coefficient $R$ if the equation (2.1) where $\lambda=k^{2}$ admits a solution $\psi$ such that :

$$
\psi=\left\{\begin{aligned}
T e^{i k x}, & \text { to the right of the barrier, } \\
e^{i k x}+R e^{-i k x}, & \text { to the left of the barrier. }
\end{aligned}\right.
$$

Theorem 2.1. a) Let $W$ be the phase plane formed by the representative points $\left(\psi, \psi^{\prime}\right)$. Let

$$
\mathscr{B}_{(2)}^{x_{1}}: E_{(2)}^{s r} \longrightarrow W, \quad \psi \longmapsto \mathscr{B}_{(2)}^{x_{1}} \psi=\left(\psi\left(x_{1}\right), \psi^{\prime}\left(x_{1}\right)\right),
$$

be an operator with $\psi$ a solution of equation (2.1) whose initial conditions for $x=x_{1} \in \mathbb{R}$ are $\left(\psi\left(x_{1}\right), \psi^{\prime}\left(x_{1}\right)\right)$. Then the space $E_{(2)}^{s r}$ is isomorphic to $W$ and the phase application of $x_{1}$ to $x_{2}$ defined by

$$
g_{x_{1}}^{x_{2}} \equiv \mathscr{B}_{(2)}^{x_{2}}\left(\mathscr{B}_{(2)}^{x_{1}}\right)^{-1}: W \longrightarrow W, \quad\left(\psi\left(x_{1}\right), \psi^{\prime}\left(x_{1}\right)\right) \longmapsto\left(\psi\left(x_{2}\right), \psi^{\prime}\left(x_{2}\right)\right),
$$

is a linear isomorphism.

b) If equation (2.1) where $\lambda=k^{2}$, has a confounded solution with a $e^{i k x}$ for $x \ll 0$ and with $b e^{-i k x}$ for $x \gg 0$, then this solution is null. In addition, for all $k>0$ the $\psi, T$ and $R$ defined above exist and are unique.

Proof. a) $\mathscr{B}_{(2)}^{x_{1}}$ is linear and for any representative point $\left(\psi, \psi^{\prime}\right) \in W$, there exists from the existence theorem (differential equations) a solution $\psi$ satisfying the initial condition $\left(\psi\left(x_{1}\right), \psi^{\prime}\left(x_{1}\right)\right)$. Then $\operatorname{Im}_{\mathscr{B}_{(2)}^{x_{1}}} \equiv\left\{\mathscr{B}_{(2)}^{x_{1}} \psi: \psi \in E_{(2)}^{s r}\right\}=W$. Finally $\operatorname{Ker} \mathscr{B}_{(2)}^{x_{1}} \equiv\left\{\psi: \psi \in E_{(2)}^{s r}, \mathscr{B}_{(2)}^{x_{1}} \psi=0\right\}=0$, follows from the uniqueness theorem because the solution satisfying the initial condition at the point $x_{1}$ is equal to zero. The result follows from the fact that the inverse of an isomorphism is one. If $\psi_{1}$ and $\psi_{2}$ are two solutions of equation (2.1), then $\left(\psi\left(x_{1}\right), \psi^{\prime}\left(x_{1}\right)\right)=\mathscr{B}_{(2)}^{x_{1}} \psi_{1}+\mathscr{B}_{(2)}^{x_{1}} \psi_{2}=\left(\psi_{1}\left(x_{1}\right), \psi_{1}^{\prime}\left(x_{1}\right)\right)+\left(\psi_{2}\left(x_{1}\right), \psi_{2}^{\prime}\left(x_{1}\right)\right)$, and this is equivalent to

$$
\left(\mathscr{B}_{(2)}^{x_{1}}\right)^{-1}\left(\left(\psi_{1}\left(x_{1}\right), \psi_{1}^{\prime}\left(x_{1}\right)\right)+\left(\psi_{2}\left(x_{1}\right), \psi_{2}^{\prime}\left(x_{1}\right)\right)\right)=\left(\mathscr{B}_{(2)}^{x_{1}}\right)^{-1}\left(\psi_{1}\left(x_{1}\right), \psi_{1}^{\prime}\left(x_{1}\right)\right)+\left(\mathscr{B}_{(2)}^{x_{1}}\right)^{-1}\left(\psi_{2}\left(x_{1}\right), \psi_{2}^{\prime}\left(x_{1}\right)\right) .
$$

b) Let be $\left\langle a e^{i k x}, a e^{i k x}\right\rangle,\left\langle b e^{-i k x}, b e^{-i k x}\right\rangle$ and $\left\langle a e^{i k x}, a e^{-i k x}\right\rangle$ the hermitian forms in the space $E_{(2)}^{s c}$. Let's designate by [.,.] the left scalar product, then

$$
\left\langle a e^{i k x}, a e^{i k x}\right\rangle=\frac{i}{2}\left[a e^{i k x}, \bar{a} e^{-i k x}\right]=\frac{i}{2}\left|\begin{array}{cc}
a & i a \\
\bar{a} & -i \bar{a}
\end{array}\right|=|a|^{2} .
$$

Similarly, we have $\left\langle b e^{-i k x}, b e^{-i k x}\right\rangle=-|b|^{2}$ et $\left\langle a e^{i k x}, a e^{-i k x}\right\rangle=0$. By setting $z=z_{1} e^{i k x}+z_{2} e^{-i k x}$ where $z_{1}$ and $z_{2}$ are the coordinates of the vector $z$ in the basis $\left(e^{i k x}, e^{-i k x}\right)$, we obtain $\langle z, z\rangle=\left|z_{1}\right|^{2}-\left|z_{2}\right|^{2}$, i.e., $\langle.,$.$\rangle is of type (1,1)$. Since the 
monodromy operator retains this hermitian form, we deduce that $|a|^{2}=-|b|^{2}$ and so $a=b=0$. Consider now a particle going to $+\infty$ and let $e^{i k x}$ be a solution to the right of the barrier. To the left of the barrier this solution becomes

$$
e^{i k x} \curvearrowright a e^{i k x}+b e^{-i k x} \text {. }
$$

From what precedes, the coefficient $a$ is nonzero. So to have the solution in question, simply divide the two members of (2.3) by $a, \frac{1}{a} e^{i k x} \curvearrowright e^{i k x}+\frac{b}{a} e^{-i k x}$. Taking $T=\frac{1}{a}$ and $R=\frac{b}{a}$, this shows that $T$ and $R$ are uniquely defined.

In the same way, we can define an operator $\mathscr{B}_{(3)}^{x_{1}}$ of $E_{(3)}^{s r}$ in $W$ that associates with each solution of equation (2.2), its initial condition at the point $x_{1}$. In this case, instead of "phase application", there will be "phase point".

We will now demonstrate a theorem that will be useful later.

Theorem 2.2. (Liouville). Let $\frac{d x}{d t}=f(x), x=\left(x_{1}, \ldots, x_{n}\right)$, be a system of ordinary differential equations whose solutions extend to the whole time axis. Let $\left\{g^{t}\right\}$ be the corresponding group of transformations : $g^{t} x=x+f(x) t+o\left(t^{2}\right)$, for $t$ small. We denote by $D$ a domain in phase space, $D(t) \equiv g^{t} D(0)$ and by $v(t)$ the volume of $D(t)$. If div $f=\sum_{j=1}^{n} \frac{\partial f_{j}}{\partial x_{j}}=0$, then $v(t)=v(0)$, i.e., $g^{t}$ preserves the volume of any domain.

Proof. We have $v(t)=\int_{D(t)} d x=\int_{D(0)} \frac{\partial g^{t} x}{\partial x} d x$, where $\frac{\partial g^{t} x}{\partial x}$ is the Jacobian matrix, $\frac{\partial g^{t} x}{\partial x}=I+\frac{\partial f}{\partial x} t+o\left(t^{2}\right)$. The determinant of the operator $I+\frac{\partial f}{\partial x} t$ is equal to the product of the eigenvalues. These (taking into account their multiplicities) are equal to $1+t \frac{\partial f_{j}}{\partial x_{j}}$ where $\frac{\partial f_{j}}{\partial x_{j}}$ are the eigenvalues of $\frac{\partial f}{\partial x}$. Then

$$
\operatorname{det} \frac{\partial g^{t} x}{\partial x}=1+t \sum_{j=1}^{n} \frac{\partial f_{j}}{\partial x_{j}}+o\left(t^{2}\right)=1+t \operatorname{div} f+o\left(t^{2}\right)
$$

Therefore, $v(t)=\int_{D(0)}\left(1+t \operatorname{div} f+o\left(t^{2}\right)\right) d x$, and $\left.\frac{d v(t)}{d t}\right|_{t=0}=\int_{D(0)} \operatorname{div} f d x$. Since $t=t_{0}$ is not worse than $t=0$, we also have

$$
\left.\frac{d v(t)}{d t}\right|_{t=t_{0}}=\int_{D\left(t_{0}\right)} \operatorname{div} f d x
$$

and the proof of the theorem follows.

Note that the Liouville's theorem is easily generalized to the case of non autonomous systems $(f=f(x, t))$. Indeed, the terms of first degree in the expression of $\frac{\partial g^{t} x}{\partial x}$ remain the same. But the terms of degree greater than one do not intervene in the proof. In other words, Liouville's theorem is a first order theorem.

Let $S L(2, \mathbb{R})$ be the real unimodular group, i.e., the set of all real $2 \times 2$ matrices with determinant one. In other words, $S L(2, \mathbb{R})$ is the group of all linear transformations of $\mathbb{R}^{2}$ that preserve oriented area $[.,$.$] (see the notation used in the proof of$ theorem 2.1). Consider the group $S U(1,1)$ of $(1,1)$-unitary unimodular matrices. This is the set of all complex $2 \times 2$ matrices with determinant one preserving the hermitian form $\left|z_{1}\right|^{2}-\left|z_{2}\right|^{2}$ (see again the notation used in the proof of theorem 2.1). In other words, they are matrices of the form $\left(\begin{array}{cc}a & b \\ c & d\end{array}\right)$ for which $|a|^{2}-|b|^{2}=|c|^{2}-|d|^{2}=1, a \bar{c}-b \bar{d}=0, a d-b c=1$.

Theorem 2.3. The matrix of the monodromy operator $\mathscr{M}$ in the basis $(\cos k x, \sin k x)\left(\right.$ resp. $\left.\left(e^{i k x}, e^{-i k x}\right)\right)$ belongs to the group $S L(2, \mathbb{R})($ resp. $S U(1,1)$ ).

Proof. We show that the determinant of the monodromy operator of the Schrödinger equation is equal to one. Note that $(\cos k x, \sin k x)$ is a basis on the space $E_{(3)}^{s r}$. As $\mathscr{B}_{(3)}^{x} \cos k x=(\cos k x,-k \sin k x)$ and $\mathscr{B}_{(3)}^{x} \sin k x=(\sin k x, k \cos k x)$, so $W$ is provided with a basis in which the matrix of the operator (we use here the same notation for the operator and the matrix) is written

$$
\mathscr{B}_{(3)}^{x}=\left(\begin{array}{cc}
\cos k x & \sin k x \\
-k \sin k x & k \cos k x
\end{array}\right)
$$


hence det $\mathscr{B}_{(3)}^{x}=k$, independent of $x$. Let us denote by $x^{+}$the point $x$ to the left of the support of the potential and by $x^{-}$the one on the right. We have the following situation :

$$
\begin{aligned}
& \mathscr{M}: E_{(3)}^{s r} \longrightarrow E_{(3)}^{s r}, \quad a \cos k x+b \sin k x \longmapsto c \cos k x+d \sin k x, \quad(c, d)=\mathscr{M}_{u}(a, b), \\
& \mathscr{B}_{(3)}^{x^{-}}: E_{(3)}^{s r} \longrightarrow W, \quad a \cos k x+b \sin k x \longmapsto\left(a \cos k x^{-}+b \sin k x^{-},-a k \sin k x^{-}+b k \cos k x^{-}\right), \\
& \mathscr{B}_{(3)}^{x^{+}}: E_{(3)}^{s r} \longrightarrow W, \quad c \cos k x+d \sin k x \longmapsto\left(a \cos k x^{+}+b \sin k x^{+},-a k \sin k x^{+}+b k \cos k x^{+}\right), \\
& g_{x^{-}}^{x^{+}}: W \longrightarrow W, \quad\left(a \cos k x^{-}+b \sin k x^{-},-a k \sin k x^{-}+b k \cos k x^{-}\right) \longmapsto\left(a \cos k x^{+}+b \sin k x^{+},-a k \sin k x^{+}+b k \cos k x^{+}\right) .
\end{aligned}
$$

We verify directly that $: g_{x^{-}}^{x^{+}} o \mathscr{B}_{(3)}^{x^{-}}=\mathscr{B}_{(3)}^{x^{+}} o \mathscr{M}$, and since $\operatorname{det} \mathscr{B}_{(3)}^{x^{+}}=\mathscr{B}_{(3)}^{x^{-}}$, so we have det $\mathscr{M}=\operatorname{det} g_{x^{-}}^{x^{+}}$. Now $g^{x}$ preserves the areas according to Liouville's theorem (indeed, by putting $\psi_{1}=\psi, \psi_{2}=\psi^{\prime}$, we rewrite equation (2.1) under form

$$
\psi_{1}^{\prime}=\psi_{2} \equiv f_{1}, \quad \psi_{2}^{\prime}=(u(x)-\lambda) \psi_{1} \equiv f_{2}
$$

Here we have $f=\left(f_{1}, f_{2}\right), t=x$ and $\left.\operatorname{div} f=\frac{\partial \psi_{2}}{\partial \psi_{1}}+\frac{\partial(u(x)-\lambda) \psi_{1}}{\partial \psi_{2}}=0\right)$. Therefore, $\operatorname{det} g_{x^{-}}^{x^{+}}=1$ and consequently det $\mathscr{M}=1$. For the case of $S U(1,1)$, we will show that the matrix (also denoted $\mathscr{M}$ ) of an operator is real and unimodular in the basis $(\cos k x, \sin k x)$ if and only if it is special $(1,1)$-unitary in complex conjugate basis $\left(e^{i k x}, e^{-i k x}\right)$. By setting as in the proof of theorem $1, z=z_{1} e^{i k x}+z_{2} e^{-i k x}$ where $z_{1}$ and $z_{2}$ are the coordinates of the vector $z$ in the basis $\left(e^{i k x}, e^{-i k x}\right)$, we obtain $\langle z, z\rangle=\left|z_{1}\right|^{2}-\left|z_{2}\right|^{2}$, i.e., $\langle.,$.$\rangle is of type (1,1)$. The monodromy operator conserves this hermitian form. Say that $\mathscr{M}$ is real and unimodular in the basis $(\cos k x, \sin k x)$ is equivalent to $\mathscr{M} \in G L(2, \mathbb{R}) \cap S L(2, \mathbb{C})$ or what amounts to the same $\mathscr{M} \in S U(1,1)$ or what is equivalent $\mathscr{M}$ is $(1,1)$-unitary and unimodular in the basis $\left(e^{i k x}, e^{-i k x}\right)$.

Define the solutions $\psi_{1}(x, \lambda)$ and $\psi_{2}(x, \lambda)$ of equation $(2.1)$ by the initial conditions : $\psi_{1}(0, \lambda)=1, \psi_{1}^{\prime}(0, \lambda)=0$, $\psi_{2}(0, \lambda)=0, \psi_{2}^{\prime}(0, \lambda)=1$. For the simple case $u(x)=0$, we obviously have

$$
\begin{aligned}
& \psi_{1}(x, \lambda)=\cos \sqrt{\lambda} x=1+\left(-\frac{1}{2} \lambda\right) x^{2}+\left(\frac{1}{24} \lambda^{2}\right) x^{4}+O\left(x^{6}\right), \\
& \psi_{2}(x, \lambda)=\frac{1}{\sqrt{\lambda}} \sin \sqrt{\lambda} x=x+\left(-\frac{1}{6} \lambda\right) x^{3}+\left(\frac{1}{120} \lambda^{2}\right) x^{5}+O\left(x^{7}\right) .
\end{aligned}
$$

For $\sqrt{\lambda}$, we can choose for example the determination $\sqrt{\lambda}=\sqrt{r} e^{i \frac{\theta}{2}}$ where $\lambda=r e^{i \theta}$ with $r>0$ and $-\pi<\theta<\pi$. Let $\alpha$ be an arbitrary real number. The function $\psi(x, \lambda)=\psi_{1}(x, \lambda)+\alpha \psi_{2}(x, \lambda)$ is also solution of equation (2.1) and satisfies the boundary condition $\psi^{\prime}(0, \lambda)-\alpha \psi(0, \lambda)=0$. For $\alpha=0$, we have $\psi(x, \lambda)=\psi_{1}(x, \lambda)$ and for $\alpha=\infty$, we put $\psi(x, \lambda)=\psi 2(x, \lambda)$. We assume that for $\lambda \in \mathbb{C}$ and $x \geq 0$, we have

$$
\psi(x, \lambda)=\cos \sqrt{\lambda} x+\int_{0}^{x} K(x, t) \cos \sqrt{\lambda} t d t
$$

where $K$ is a function to be determined, subject to the condition of having partial derivatives of order one and order two continuous in the set of real pairs $(x, t)$ such that : $0 \leq t \leq x$. In other words, we look for $\psi(x,$.$) as a perturbation of the function$ $x \mapsto \psi(x, \lambda)=\cos \sqrt{\lambda} x$ and precisely, as a transform $(I+K) \psi_{1}(x,$.$) where K$ is a Volterra operator in $[0,+\infty[$. We will look for the conditions that $K(x, t)$ must satisfy for the function (2.5) to be a solution of the differential equation (2.1). From equation (2.5), we get

$$
\frac{\partial^{2} \psi}{\partial x^{2}}(x, \lambda)=-\lambda \cos \sqrt{\lambda} x+\frac{d K(x, x)}{d x} \cos \sqrt{\lambda} x-\sqrt{\lambda} K(x, x) \sin \sqrt{\lambda} x+\left.\frac{\partial K(x, t)}{\partial x}\right|_{t=x} \cos \sqrt{\lambda} x+\int_{0}^{x} \frac{\partial^{2} K(x, t)}{\partial x^{2}} \cos \sqrt{\lambda} t d t
$$

Let's calculate the expression $\lambda \int_{0}^{x} K(x, t) \cos \sqrt{\lambda} t d t$, by doing two integrations in parts, we get

$$
\lambda \int_{0}^{x} K(x, t) \cos \sqrt{\lambda} t d t=\sqrt{\lambda} K(x, x) \sin \sqrt{\lambda} x+\left.\frac{\partial K(x, t)}{\partial t}\right|_{t=x} \cos \sqrt{\lambda} x-\left.\frac{\partial K(x, t)}{\partial t}\right|_{t=0}-\int_{0}^{x} \frac{\partial^{2} K(x, t)}{\partial t^{2}} \cos \sqrt{\lambda} t d t
$$


To calculate expression (2.1), substitute (2.6) and (2.7),

$$
\begin{aligned}
0= & \psi^{\prime \prime}+(\lambda-u(x)) \psi \\
= & \frac{d K(x, x)}{d x} \cos \sqrt{\lambda} x+\left(\frac{\partial K(x, t)}{\partial t}+\frac{\partial K(x, t)}{\partial x}\right)_{x=t} \cos \sqrt{\lambda} x-\left.\frac{\partial K(x, t)}{\partial t}\right|_{t=0}-u(x) \cos \sqrt{\lambda} x \\
& +\int_{0}^{x}\left(\frac{\partial^{2} K(x, t)}{\partial x^{2}}-\frac{\partial^{2} K(x, t)}{\partial t^{2}}-u(x) K(x, t)\right) \cos \sqrt{\lambda} t d t .
\end{aligned}
$$

We have

$$
\frac{\partial^{2} K(x, t)}{\partial x^{2}}-u(x) K(x, t)=\frac{\partial^{2} K(x, t)}{\partial t^{2}}
$$

with the boundary conditions

$$
\begin{aligned}
& \left.\frac{\partial K(x, t)}{\partial t}\right|_{t=0}=0, \\
& \frac{d K(x, x)}{d x}=\frac{1}{2} u(x) .
\end{aligned}
$$

For the initial conditions, we have $\psi(0, \lambda)=1$ and $\psi^{\prime}(0, \lambda)=K(0,0)$. As $\psi^{\prime}(0, \lambda)-\alpha \psi(0, \lambda)=0$, then $K(0,0)=\alpha$. Therefore,

$$
K(x, x)=\alpha+\frac{1}{2} \int_{0}^{x} u(t) d t .
$$

If $u(x)$ has a continuous derivative, then there exists a unique solution of (2.8), satisfying conditions (2.9) and (2.11). Hence, there exists a satisfying function $K(x, t)(2.5)$. Let's solve equation (2.5) as an equation of Volterra, we get

$$
\cos \sqrt{\lambda} x=\psi(x, \lambda)-\int_{0}^{x} K_{1}(x, t) \psi(t, \lambda) d t
$$

and in the same way as before, we show that $K_{1}(x, t)$ is solution of the equation

$$
\frac{\partial^{2} K_{1}(x, t)}{\partial x^{2}}=\frac{\partial^{2} K_{1}(x, t)}{\partial t^{2}}-u(t) K_{1}(x, t),
$$

with the conditions $\left(\frac{\partial K_{1}}{\partial t}-\alpha K_{1}\right)_{t=0}=0, K_{1}(x, x)=\alpha+\frac{1}{2} \int_{0}^{x} u(t) d t$.

For the case $\alpha=\infty$, we look for $\psi(x, \lambda)$ as a perturbation of the function $x \longmapsto \psi(x, \lambda)=\frac{1}{\sqrt{\lambda}} \sin \sqrt{\lambda} x$ (see expression (2.4)) or what is equivalent as a transform $(I+K) \psi_{1}(x,$.$) where K$ is a Volterra operator in $[0,+\infty[$. In other words, we set $\lambda \in \mathbb{C}$ and $x \geq 0$,

$$
\psi(x, \lambda)=\frac{\sin \sqrt{\lambda} x}{\sqrt{\lambda}}+\int_{0}^{x} L(x, t) \frac{\sin \sqrt{\lambda} x}{\sqrt{\lambda} t} d t,
$$

where $L$ is a function to be determined, subject to the condition of having partial derivatives of order one and order two continuous in the set of real pairs $(x, t)$ such that : $0 \leq t \leq x$. By reasoning as before, we obtain the relation

$$
\frac{\partial^{2} L(x, t)}{\partial x^{2}}-u(x) L(x, t)=\frac{\partial^{2} L(x, t)}{\partial t^{2}},
$$

with the conditions $L(x, x)=0, L(x, x)=\frac{1}{2} \int_{0}^{x} u(t) d t$. By solving equation (2.13), we obtain

$$
\frac{\sin \sqrt{\lambda} x}{\sqrt{\lambda}}=\psi(x, \lambda)+\int_{0}^{x} L_{1}(x, t) \psi(t, \lambda) d t
$$


The functions $L(x, t)$ and $L_{1}(x, t)$ have the same properties as the functions $K(x, t)$ and $K_{1}(x, t)$ previously obtained.

Recall that for every function $f \in L^{2}(\mathbb{R})$, we have the Parseval identity $\int_{0}^{\infty} f^{2}(x) d x=\int_{-\infty}^{\infty} F^{2}(\lambda) \cdot d \rho(\lambda)$ where $\mathscr{F}\{f(x)\} \equiv$ $F(\lambda)=\int_{0}^{\infty} f(x) \psi(x, \lambda) d x$ is the Fourier transform of $f(x)$ and $\rho(\lambda)$ a monotone function, bounded on any finite interval. The sequence of functions $F_{n}(\lambda)=\int_{0}^{n} f(x) \psi(x, \lambda) d x$, converges in quadratic mean (with respect to the spectral measure $\left.\rho(\lambda)\right)$ to $F(\lambda)$, i.e., $\lim _{n \rightarrow \infty} \int_{-\infty}^{\infty}\left(F(\lambda)-F_{n}(\lambda)\right)^{2} d \rho(\lambda)=0$. We choose $\rho(\lambda)$ in the following form $: \rho(\lambda)=\frac{2}{\pi} \sqrt{\lambda}+\sigma(\lambda)$ if $\lambda>0$ and $\rho(\lambda)=\sigma(\lambda)$ if $\lambda<0$, where $\sigma(\lambda)$ is a measure with compact support satisfying the condition : $\int_{-\infty}^{\infty}|\lambda| .|d \sigma(\lambda)|<+\infty$. For $0<b<y<a<x$, the functions $\int_{a}^{x} \psi(t, \lambda) d t$ and $\int_{b}^{y} \cos \sqrt{\lambda} t d t$ are orthogonal with respect to $\rho(\lambda)$. In other words, we have the orthogonality relation :

$$
I \equiv \int_{-\infty}^{\infty}\left(\int_{a}^{x} \psi(t, \lambda) d t\right)\left(\int_{b}^{y} \cos \sqrt{\lambda} t d t\right) d \rho(\lambda)=0 .
$$

Indeed, by integrating equation (2.12) from $b$ to $y$, we obtain

$$
\begin{aligned}
\int_{b}^{y} \cos \sqrt{\lambda} t d t & =\int_{b}^{y} \psi(t, \lambda) d t-\int_{b}^{y} d t \int_{0}^{t} K_{1}(t, s) \psi(s, \lambda) d s \\
& =\int_{b}^{y} \psi(t, \lambda) d t-\int_{0}^{b} \psi(s, \lambda) d s \int_{b}^{y} K_{1}(t, s) d t-\int_{b}^{y} \psi(s, \lambda) d t \int_{s}^{y} K_{1}(t, s) d t .
\end{aligned}
$$

By definition, this function is expressed using the transform (in $\psi(t, \lambda)$ ) of a null function outside the interval $] b, y[$. Since ]$b, y[\cap] a, x[=\emptyset$, we deduce from Parseval's equality that we have $I=0$.

To obtain the Gelfand-Levitan integral equation [4, 5], we proceed as follows: according to equation (2.5), we have

$$
\begin{aligned}
\int_{a}^{x} \psi(t, \lambda) d t & =\int_{a}^{x} \cos \sqrt{\lambda} t d t+\int_{a}^{x} d t \int_{0}^{t} K(t, s) \cos \sqrt{\lambda} s d s \\
& =\int_{a}^{x} \cos \sqrt{\lambda} t d t+\int_{0}^{a} \cos \sqrt{\lambda} s d s \int_{a}^{x} K(t, s) d t+\int_{a}^{x} \cos \sqrt{\lambda} s d s \int_{s}^{x} K(t, s) d t
\end{aligned}
$$

by virtue of Lebesgue-Fubini's theorem. Therefore,

$$
\begin{aligned}
I= & \int_{-\infty}^{\infty}\left(\int_{a}^{x} \cos \sqrt{\lambda} t d t\right)\left(\int_{b}^{y} \cos \sqrt{\lambda} t d t\right) d \rho(\lambda) \\
& +\int_{-\infty}^{\infty}\left(\int_{0}^{a} \cos \sqrt{\lambda} s d s \int_{a}^{x} K(t, s) d t+\int_{a}^{x} \cos \sqrt{\lambda} s d s \int_{s}^{x} K(t, s) d t\right) \times\left(\int_{b}^{y} \cos \sqrt{\lambda} t d t\right) d \rho(\lambda)=0 .
\end{aligned}
$$

This expression can be written using the definition of $\rho(\lambda)$, in the form

$$
\begin{aligned}
I= & \int_{-\infty}^{\infty}\left(\int_{a}^{x} \cos \sqrt{\lambda} t d t\right)\left(\int_{b}^{y} \cos \sqrt{\lambda} t d t\right) d \sigma(\lambda) \\
& +\int_{-\infty}^{\infty}\left(\int_{0}^{a} \cos \sqrt{\lambda} s d s \int_{a}^{x} K(t, s) d t+\int_{a}^{x} \cos \sqrt{\lambda} s d s \int_{s}^{x} K(t, s) d t\right) \times\left(\int_{b}^{y} \cos \sqrt{\lambda} t d t\right) d \sigma(\lambda) \\
& +\frac{2}{\pi} \int_{-\infty}^{\infty}\left(\int_{a}^{x} \cos \sqrt{\lambda} t d t\right)\left(\int_{b}^{y} \cos \sqrt{\lambda} t d t\right) d \sigma(\lambda) \\
& +\frac{2}{\pi} \int_{-\infty}^{\infty}\left(\int_{0}^{a} \cos \sqrt{\lambda} s d s \int_{a}^{x} K(t, s) d t+\int_{a}^{x} \cos \sqrt{\lambda} s d s \int_{s}^{x} K(t, s) d t\right) \times\left(\int_{b}^{y} \cos \sqrt{\lambda} t d t\right) d \sigma(\lambda)=0
\end{aligned}
$$

Since $b<y<a<x$, then given the Parseval identity, the third term is equal to zero while the fourth is equal to

$$
\int_{b}^{y}\left(\int_{0}^{a} \cos \sqrt{\lambda} s d s \int_{a}^{x} K(t, s) d t+\int_{a}^{x} \cos \sqrt{\lambda} s d s \int_{s}^{x} K(t, s) d t\right) d s=\int_{b}^{y} d s \int_{a}^{x} K(t, s) d t .
$$


Therefore,

$$
\begin{aligned}
I= & \int_{-\infty}^{\infty} \frac{(\sin \sqrt{\lambda} x-\sin \sqrt{\lambda} a)(\sin \sqrt{\lambda} y-\sin \sqrt{\lambda} b)}{\lambda} d \sigma(s) \\
& +\int_{-\infty}^{\infty}\left(\int_{0}^{a} \cos \sqrt{\lambda} s d s \int_{a}^{x} K(t, s) d t+\int_{a}^{x} \cos \sqrt{\lambda} s d s \int_{s}^{x} K(t, s) d t\right) \times\left(\int_{b}^{y} \cos \sqrt{\lambda} s d s\right) d \sigma(\lambda) \\
& +\int_{b}^{y} d s \int_{a}^{x} K(t, s) d t=0 .
\end{aligned}
$$

By setting

$$
F(x, y) \equiv \int_{-\infty}^{\infty} \frac{\sin \sqrt{\lambda} x \sin \sqrt{\lambda} y}{\lambda} d \sigma(\lambda)
$$

and

$$
G(x, s) \equiv\left\{\begin{aligned}
\int_{a}^{x} K(t, s) d t, & 0 \leq s \leq a \\
\int_{s}^{x} K(t, s) d t, & a \leq s \leq x \\
0, & s>x
\end{aligned}\right.
$$

the equation above becomes

$$
F(x, y)-F(x, b)-F(a, y)+F(a, b)+\int_{b}^{y} d s \int_{a}^{x} K(t, s) d t+\int_{-\infty}^{\infty}\left(\int_{0}^{x} G(x, s) \cos \sqrt{\lambda} s d s\right)\left(\int_{b}^{y} \cos \sqrt{\lambda} s d s\right) d \sigma(\lambda)=0 .
$$

This last equation can still be written, doing an integration by parts and noticing that $G(x, x)=0$,

$$
F(x, y)-F(x, b)-F(a, y)+F(a, b)+\int_{b}^{y} d s \int_{a}^{x} K(t, s) d t+\int_{-\infty}^{\infty}\left(\int_{0}^{x} \frac{\partial G(x, s)}{\partial s} \frac{\sin \sqrt{\lambda} s}{\sqrt{\lambda}} d s\right)\left(\frac{\sin \sqrt{\lambda} y-\sin \sqrt{\lambda} b}{\sqrt{\lambda}}\right) d \sigma(\lambda)=0 .
$$

But

$$
\begin{aligned}
& \int_{-\infty}^{\infty}\left(\int_{0}^{x} \frac{\partial G(x, s)}{\partial s} \frac{\sin \sqrt{\lambda} s}{\sqrt{\lambda}} d s\right)\left(\frac{\sin \sqrt{\lambda} y-\sin \sqrt{\lambda} b}{\sqrt{\lambda}}\right) d \sigma(\lambda) \\
& =\int_{0}^{x} \frac{\partial G(x, s)}{\partial s}\left(\int_{-\infty}^{\infty}\left(\frac{\sin \sqrt{\lambda} s \sin \sqrt{\lambda} y-\sin \sqrt{\lambda} s \sin \sqrt{\lambda} b}{\lambda}\right) d \sigma(\lambda)\right) d s \\
& =\int_{0}^{x} \frac{\partial G(x, s)}{\partial s}(F(s, y)-F(s, b)) d s \\
& =-\int_{0}^{x} G(x, s)\left(\frac{\partial F(s, y)}{\partial s}-\frac{\partial F(s, b)}{\partial s}\right) d s \\
& =-\int_{0}^{a}\left(\frac{\partial F(s, y)}{\partial s}-\frac{\partial F(s, b)}{\partial s}\right) d s\left(\int_{a}^{x} K(t, s) d t\right)-\int_{a}^{x}\left(\frac{\partial F(s, y)}{\partial s}-\frac{\partial F(s, b)}{\partial s}\right) d s\left(\int_{s}^{x} K(t, s) d t\right), \\
& =\int_{a}^{x} d t \int_{0}^{t}\left(\frac{\partial F(s, y)}{\partial s}-\frac{\partial F(s, b)}{\partial s}\right) d s
\end{aligned}
$$

so equation (2.15) becomes

$$
F(x, y)-F(x, b)-F(a, y)+F(a, b)+\int_{b}^{y} d s \int_{a}^{x} K(t, s) d t+\int_{a}^{x} d t \int_{0}^{t}\left(\frac{\partial F(s, y)}{\partial s}-\frac{\partial F(s, b)}{\partial s}\right) d s=0 .
$$

Deriving this expression with respect to $y$ and then with respect to $x$ (the support of the measure $\sigma$ is compact), we obtain

$$
\frac{\partial^{2} F}{\partial x \partial y}+\int_{0}^{x} K(x, s) \frac{\partial^{2} F(s, y)}{\partial s \partial y}+K(x, y)=0 .
$$


By setting $f(x, y) \equiv \frac{\partial^{2} F}{\partial x \partial y}$, we finally obtain the Gelfand-Levitan integral equation for the function $x \longmapsto K(x, y)$ valid for $0<y<x$,

$$
f(x, y)+K(x, y)+\int_{0}^{x} K(x, s) f(s, y) d s=0, \quad y \leq x .
$$

For the case $\alpha=\infty$, i.e., $\psi(x, \lambda)=\psi_{2}(x, \lambda)$, just integrate the two members of equation (2.14) from 0 to $x$ and use a similar reasoning. Under the continuity assumption of $K$, equation (2.16) must be checked for $x=0$ and $x=y$. Note also that if we set $x$ in the previous equation, then we will obtain the so called Fredholm's linear integral equation. We can prove that, conversely, equation (2.16) admits a single continuous solution in the set of pairs of real numbers such that : $0 \leq t \leq x$. We will not look for the solution at this level, it will be done later (in the next section) when we treat the Korteweg-de-Vries equation.

\section{KdV equation and the inverse diffusion method}

Let us first examine some particular solutions of the equation of $\mathrm{KdV}(1.1)$, of the kind of progressive waves $u(x, t)=s(x-c t)$, where $c$ is the phase velocity. By replacing this expression in (1.1), we obtain $-c \frac{\partial s}{\partial x}-6 s \frac{\partial s}{\partial x}+\frac{\partial^{3} s}{\partial x^{3}}=0$. By integrating this equation with respect to $x$ and imposing the boundary condition that $s$ and its derivatives decrease for $|x| \longrightarrow \infty$, we get $-c s-3 s^{2}+\frac{\partial^{2} s}{\partial x^{2}}=0$, hence $-c s-2 s^{3}+\left(\frac{\partial s}{\partial x}\right)^{2}=0$, and the exact expression of the solution $s$ requires the use of elliptic functions. Suppose that $\frac{\partial s}{\partial x}(0)=0$, in which case the solution of this last equation is $s(x-c t)=-\frac{c}{2} \operatorname{sech}^{2} \frac{\sqrt{c}}{2}(x-c t)$, where sech denotes the hyperbolic secant, i.e., $\frac{1}{\cosh }$. Therefore $u(x, 0)=u_{0} \operatorname{sech}^{2} \frac{x}{l}$, where $u_{0} \equiv-\frac{c}{2}$ et $l^{2} \equiv \frac{4}{c}$. This expression shows that $u$ removes infinitely long time in the position $u \simeq 0$, then it reaches the value $u_{0}$, is reflected on this point and returns again in the position of $u \simeq 0$. This solution is called soliton. To obtain this solution, we can use the so-called Bäcklund transformations for the Korteweg de Vries equation.

When solitons collide, dimensions and speeds of solutions do not change after collision. This phenomenon has suggested the idea of conservation laws. And indeed, Kruskal, Zabusky, Lax, Gardner, Green and Miura [6, 7, 8, 9, 10, 11, 12, 13, 14] have been able to find a whole series of first integrals for the $\mathrm{KdV}$ equation. These integrals are of the form $\int P_{n}\left(u, \ldots, u^{(n)}\right) d x$, where $P_{n}$ is a polynomial. Indeed, the conservation equations that can be deduced from the KdV equation take the following general form : $\frac{\partial P_{n}}{\partial t}+\frac{\partial Q_{n}}{\partial x}=0$, where $P_{n}$ and $Q_{n}$ form a series of functions of which here are the first three:

(i) The KdV equation can be written in the form

$$
\frac{\partial u}{\partial t}+\frac{\partial}{\partial x}\left(-3 u^{2}+\frac{\partial^{2} u}{\partial x^{2}}\right)=0 \Longrightarrow P_{1}=u, \quad Q_{1}=-3 u^{2}+\frac{\partial^{2} u}{\partial x^{2}}
$$

(ii) Multiply the $\mathrm{KdV}$ equation by $u$, this gives

$$
u \frac{\partial u}{\partial t}-6 u^{2} \frac{\partial u}{\partial x}+u \frac{\partial^{3} u}{\partial x^{3}}=0, \quad \frac{\partial}{\partial t}\left(\frac{u^{2}}{2}\right)+\frac{\partial}{\partial x}\left(-2 u^{3}+u \frac{\partial^{2} u}{\partial x^{2}}-\frac{1}{2}\left(\frac{\partial u}{\partial x}\right)^{2}\right)=0 .
$$

Hence,

$$
P_{2}=\frac{u^{2}}{2}, \quad Q_{2}=-2 u^{3}+u \frac{\partial^{2} u}{\partial x^{2}}-\frac{1}{2}\left(\frac{\partial u}{\partial x}\right)^{2}
$$

(iii) We have

$$
\begin{aligned}
& \left(3 u^{2}-\frac{\partial^{2} u}{\partial x^{2}}\right)\left(\frac{\partial u}{\partial t}-6 u \frac{\partial u}{\partial x}+\frac{\partial^{3} u}{\partial x^{3}}\right)=0 \\
& \left(3 u^{2} \frac{\partial u}{\partial t}+\frac{\partial u}{\partial x} \frac{\partial^{2} u}{\partial x \partial t}\right)+\left(-18 u^{3} \frac{\partial u}{\partial x}+3 u^{2} \frac{\partial^{3} u}{\partial t^{3}}+6 u \frac{\partial u}{\partial x} \frac{\partial^{2} u}{\partial x^{2}}-\frac{\partial^{2} u}{\partial x^{2}} \frac{\partial^{3} u}{\partial x^{3}}-\frac{\partial^{2} u}{\partial x^{2}} \frac{\partial u}{\partial t}-\frac{\partial u}{\partial x} \frac{\partial^{2} u}{\partial x \partial t}\right)=0
\end{aligned}
$$

Therefore,

$$
\frac{\partial}{\partial t}\left(u^{3}+\frac{1}{2}\left(\frac{\partial u}{\partial x}\right)^{2}\right)+\frac{\partial}{\partial t}\left(-\frac{9}{2} u^{4}+3 u^{2} \frac{\partial^{2} u}{\partial x^{2}}-\frac{1}{2}\left(\frac{\partial^{2} u}{\partial x^{2}}\right)^{2}-\frac{\partial u}{\partial x} \frac{\partial u}{\partial t}\right)=0 .
$$


Consequently,

$$
P_{3}=u^{3}+\frac{1}{2}\left(\frac{\partial u}{\partial x}\right)^{2}, \quad Q_{3}=-\frac{9}{2} u^{4}+3 u^{2} \frac{\partial^{2} u}{\partial x^{2}}-\frac{1}{2}\left(\frac{\partial^{2} u}{\partial x^{2}}\right)^{2}-\frac{\partial u}{\partial x} \frac{\partial u}{\partial t} .
$$

If $u$ vanishes for $x \rightarrow \infty$, we get $\frac{\partial}{\partial t} \int P_{n} d x=0$, then $\int P_{n} d x$ are first integrals of the KdV equation. Let $u(x, t)=\frac{\partial y}{\partial x}(x, t)$ and suppose that $\frac{\partial y}{\partial t}, \frac{\partial y}{\partial x}, \frac{\partial^{3} y}{\partial t^{3}}$ decay when $|x| \rightarrow \infty$. The $\mathrm{KdV}$ equation, is written

$$
\frac{\partial y}{\partial t}-3\left(\frac{\partial y}{\partial x}\right)^{2}+\frac{\partial^{3} y}{\partial t^{3}}=0
$$

Hence,

$$
\frac{\partial}{\partial t} \int_{-\infty}^{\infty} y(x, t) d x=3 \int_{-\infty}^{\infty}\left(\frac{\partial y}{\partial x}\right)^{2}(x, t) d x=3 \int_{-\infty}^{\infty} u^{2}(x, t) d x=\text { constant. }
$$

Since $u=\frac{\partial y}{\partial x}$, we have also

$$
\frac{\partial}{\partial t} \int_{-\infty}^{\infty} y(x, t) d x=\frac{\partial}{\partial t} \int_{-\infty}^{\infty} \int_{-\infty}^{x} u(z, t) d z d x=\left.x \frac{\partial}{\partial t} \int_{-\infty}^{x} u(z, t) d z\right|_{-\infty} ^{\infty}-\frac{\partial}{\partial t} \int_{-\infty}^{\infty} u(z, t) d x=-\frac{\partial}{\partial t} \int_{-\infty}^{\infty} x u(x, t) d x,
$$

because by hypothesis $u^{2}$ and $\frac{\partial^{2} u}{\partial x^{2}}$ tend to 0 when $|x| \rightarrow \infty$. Comparing the two expressions obtained, we obtain a new first integral

$$
\frac{\partial}{\partial t} \int_{-\infty}^{\infty} x u(x, t) d x=\text { constante. }
$$

Lax [7] showed that the equation of $\mathrm{KdV}$ is equivalent to the equation : $\frac{d A}{d t}=[A, B]$, where

$$
A=-\frac{\partial^{2}}{\partial x^{2}}+u(x, t), \quad B=4 \frac{\partial^{3}}{\partial x^{3}}-3\left(u \frac{\partial}{\partial x}+\frac{\partial u}{\partial x}\right)
$$

We deduce that the spectrum of $A$ is conserved : if $A$ is a symmetric operator $\left(A^{\top}=A\right)$ and $T$ an orthogonal transformation $\left(T^{\top}=T^{-1}\right)$, then the spectrum of $T^{-1} A T$ coincides with that of $A$. The appearance of an infinite series of first integrals is easily explained by the Lax equation.

The Sturm-Liouville equation $A \psi=\lambda \psi$, where $\lambda$ is a real parameter, can be written in the form

$$
\frac{\partial^{2} \psi}{\partial x^{2}}+(\lambda-u(x, t)) \psi=0 .
$$

This equation reminds us the stationary Schrödinger equation. We will see that the complete solution of the KdV equation is closely related to the solution of this equation. We will look at solutions for which $u$ decreases fast enough for $x \longrightarrow \pm \infty$. There are other interesting conditions to know: the case where $u(x, t)$ tends to different constants for $|x| \longrightarrow \infty$ and the one where $u(x, t)$ is periodic in $x$. So consider equation (3.1) where $u(x, t)$ is the solution of the $\mathrm{KdV}$ equation (1.1). It is assumed that after a certain time equation (3.1) has $N$ bound states with energy $\lambda_{n}=-k_{n}^{2}, n=1,2, \ldots, N$ and continuous states with for energy $\lambda=k^{2}$. We draw $u$ from equation (3.1) and replace it in equation (1.1). After a long calculation, after multiplying by $\psi^{2}$, we get the expression

$$
\frac{\partial \lambda}{\partial t} \cdot \psi^{2}+\frac{\partial}{\partial x}\left(\psi \frac{\partial \Upsilon}{\partial x}-\frac{\partial \psi}{\partial x} \Upsilon\right)=0
$$

where $\Upsilon \equiv \frac{\partial \psi}{\partial t}+\frac{\partial^{3} \psi}{\partial x^{3}}-3(u+\lambda) \frac{\partial \psi}{\partial x}$. 
Theorem 3.1. a) For the study of the discrete part of the spectrum $\lambda_{n}(t)=-k_{n}^{2}(t)$, we consider $\psi_{n}$ (measurable and square integrable function) and we show that if $\psi_{n}$ and $\frac{\partial \psi_{n}}{\partial x}$ tend to zeros when $|x|$ goes to infinity, then $\lambda_{n}(t)=$ constant and the solution of equation (3.1) is given by $\psi_{n}(t)=c_{n}(0) e^{k_{n}\left(x-4 k_{n}^{2} t\right)}$, where $c_{n}(0)$ is determined by the initial condition $u(x, 0)=u_{0}(x)$ of the KdV equation.

b) For the study of the continuous part of the spectrum $\lambda(t)=k^{2}(t)$, we assume that a stationary plane wave propagates from $x=-\infty$ and meets a potential $u(x, t)$ with a transmission coefficient $T$ and a reflection coefficient $R$. In this case equation (3.1) admits a solution $\psi$ such that :

$$
\psi=\left\{\begin{array}{cl}
T(k, t) e^{i k x}, & x \rightarrow+\infty(\text { i.e., to the right of the potential barrier }) \\
e^{i k x}+R(k, t) e^{-i k x}, & x \rightarrow+-\infty(i . e ., \text { to the left of the potential barrier })
\end{array}\right.
$$

where $|R|^{2}+|T|^{2}=1$. If $u \simeq 0$ for $|x| \rightarrow \infty$, then we have $T(k, t)=T(k, 0)$ and $R(k, t)=R(k, 0) e^{-8 i k^{3} t}$ where $R(k, 0)$ and $T(k, 0)$ are determined by the initial condition $u(x, 0)=u_{0}(x)$ of the KdV equation.

Proof. a) Just integrate equation (3.2), this gives

$$
\frac{\partial \lambda_{n}}{\partial t} \cdot \int_{-\infty}^{\infty} \psi_{n}^{2} d x+\psi_{n} \frac{\partial \Upsilon}{\partial x}-\frac{\partial \psi_{n}}{\partial x} \Upsilon=0
$$

By hypothesis, $\psi_{n} \in L^{2}$ and $\psi_{n}, \frac{\partial \psi_{n}}{\partial x}$ tend to zeros when $|x|$ goes to infinity, so $\psi_{n} \frac{\partial \Upsilon}{\partial x}-\frac{\partial \psi_{n}}{\partial x} \Upsilon$ tends to 0 for $|x| \rightarrow \infty$ and we deduce that $\lambda_{n}(t)=$ constant. Now, since $\frac{\partial \lambda}{\partial t}=0$, then equation (3.2) becomes $\frac{\partial}{\partial x}\left(\psi \frac{\partial \Upsilon}{\partial x}-\frac{\partial \psi}{\partial x} \Upsilon\right)=0$. Let's integrate this expression twice, $\frac{\left(\psi \frac{\partial \Upsilon}{\partial x}-\frac{\partial \psi}{\partial x} \Upsilon\right)}{\psi^{2}}=\frac{A}{\psi^{2}}$, i.e., $\left(\frac{\Upsilon}{\psi}\right)^{\prime}=\frac{A}{\psi^{2}}$, hence, $\Upsilon=\psi \int \frac{A(t)}{\psi^{2}} d x+B(t) \psi$, where $A(t)$ and $B(t)$ are integration constants. So we have

$$
\frac{\partial \psi_{n}}{\partial t}+\frac{\partial^{3} \psi_{n}}{\partial x^{3}}-3\left(u+\lambda_{n}\right) \frac{\partial \psi_{n}}{\partial x}=\psi_{n} \int \frac{A_{n}}{\psi_{n}^{2}} d x+B_{n} \psi_{n}
$$

Note that $A_{n}(t)=0$ because $\psi_{n}$ satisfies (3.3) and decreases to zeros for $t \rightarrow-\infty$. Let's consider $u \cong 0$ for $x \rightarrow-\infty$ because otherwise $\psi_{n}$ would not have the decay assumption. Multiply (3.3) by $\psi_{n}$ and integrate

$$
\int_{-\infty}^{\infty} \psi_{n} \frac{\partial \psi_{n}}{\partial t} d x+\int_{-\infty}^{\infty}\left(\psi_{n} \frac{\partial^{3} \psi_{n}}{\partial x^{3}}-3 \lambda_{n} \psi_{n} \frac{\partial \psi_{n}}{\partial x}\right) d x=B_{n} \int_{-\infty}^{\infty} \psi_{n}^{2} d x
$$

This expression can be written, by adding and subtracting $\frac{\partial \psi_{n}}{\partial x} \frac{\partial^{2} \psi_{n}}{\partial x^{2}}$,

$$
\int_{-\infty}^{\infty} \frac{1}{2} \frac{\partial \psi_{n}^{2}}{\partial t} d x+\int_{-\infty}^{\infty} \frac{\partial}{\partial x}\left(\psi_{n} \frac{\partial^{2} \psi_{n}}{\partial x^{2}}-\frac{3}{2} \lambda_{n} \psi_{n}^{2}-\frac{1}{2}\left(\frac{\partial \psi_{n}}{\partial x}\right)^{2}\right) d x=B_{n} \int_{-\infty}^{\infty} \psi_{n} d x
$$

We have $B_{n}(t)=0$ because $\psi_{n} \in L^{2}$ and decreases to zeros when $x \rightarrow-\infty$. Since $u \cong 0$ for $x \rightarrow-\infty$, then from equation (3.2), it comes $\psi_{n}(x, t)=c_{n}(t) e^{k_{n} x}, x \rightarrow-\infty$. By replacing the latter in equation (3.3), we obtain $\left(\frac{\partial c_{n}}{\partial t}+4 c_{n} k_{n}^{3}\right) e^{k_{n} x}=0$, hence $c_{n}(t)=c_{n}(0) e^{-4 k_{n}^{3} t}$. Consequently, $\psi_{n}(x, t)=c_{n}(0) e^{k_{n}\left(x-4 k_{n}^{2} t\right)}$.

b) Choose $\lambda=$ constant since the spectrum for $\lambda>0$ is continuous. So equation (3.3) remains valid,

$$
\frac{\partial \psi}{\partial t}+\frac{\partial^{3} \psi}{\partial x^{3}}-3(u+\lambda) \frac{\partial \psi}{\partial x}=\psi \int \frac{A}{\psi^{2}} d x+B \psi
$$

For $u \cong 0$, when $x \rightarrow+\infty$, we replace $\psi=T(k, t) e^{i k x}, \lambda=k^{2}$ in equation (3.4) and we get $\frac{\partial T}{\partial t}-4 i k^{3} T=\frac{A}{T} \int e^{-2 i k x} d x+B T$. For this equation to preserve meaning when $x \rightarrow+\infty$, we must have $A=0$, hence

$$
\frac{\partial T}{\partial t}-\left(4 i k^{3}+B\right) T=0
$$


Similarly, for $u \cong 0$, when $x \rightarrow-\infty$, we replace $\psi=e^{i k x}+R(k, t) e^{-i k x}, \lambda=k^{2}$ in equation (3.4) and we get

$$
\left(\frac{\partial R}{\partial t}+4 i k^{3} R-B R\right) e^{-i k x}-\left(4 i k^{3}+B\right) e^{i k x}=A\left(e^{i k x}+R e^{-i k x}\right) \int \frac{d x}{e^{2 i k x}+R^{2} e^{-2 i k x}+2 R} .
$$

For $x \rightarrow+\infty$, the equation above preserves a sense if $A=0$ and is written

$$
\left(\frac{\partial R}{\partial t}+4 i k^{3} R-B R\right) e^{-i k x}-\left(4 i k^{3}+B\right) e^{i k x}=0
$$

For $4 i k^{3}+B=0$, equation (3.5) implies that $T(k, t)=T(k, 0)$ while the condition $\frac{\partial R}{\partial t}+4 i k^{3} R-B R=0$, gives us $R(k, t)=$ $R(k, 0) e^{-8 i k^{3} t}$.

The knowledge of $c_{n}(t), k_{n}(t), n=1,2, \ldots, N$ and $R(k, t)$ allows to express $u(x, t)$ for any time; it is the problem of the inverse diffusion. The latter is reduced to the solution $K(x, y ; t)$ (to simplify the notations, the reader can obviously use $K(x, y)$ instead of $K(x, y ; t))$, of the Gelfand-Levitan linear integral equation :

$$
K(x, y ; t)+I(x+y, t)+\int_{-\infty}^{x} I(y+z, t) K(x, z ; t) d z=0, \quad y \leq x
$$

where

$$
I(x+y, t)=\frac{1}{2 \pi} \int_{-\infty}^{\infty} R(k, t) e^{-i k(x+y)} d k+\sum_{n=1}^{N} c_{n}^{2}(t) e^{k_{n}(t)(x+y)} .
$$

The solution $u(x, t)$ of the $\mathrm{KdV}$ equation is then given (see (2.10)) by

$$
u(x, t)=2 \frac{d}{d x} K(x, x ; t) .
$$

The nonlinear KdV equation is transformed into the linear Gelfand-Levitan equation. The initial problem is thus completely solved. This method presents two major simplifications. First, in the analytical approach of the solution of the KdV equation, it suffices at each stage to solve only linear equations. Then $t$ only appears parametrically and more than for all $t$ the Gelfand-Levitan equation seems superficially to be an integral equation of two variables, actually $x$ intervenes as a parameter and so we have to do to a family of integral equations for the functions $K(x, y)$ of a single variable $y$. Before dealing with the general case, i.e., the case of distinct $N$ solitons, let us return first to the case of a soliton and therefore consider the solution $u(x, t)=-\frac{c}{2} \operatorname{sech}^{2} \frac{\sqrt{c}}{2}(x-c t)$ of the $\mathrm{KdV}$ equation obtained previously with the following initial condition : $u(x, 0)=-2 \operatorname{sech}^{2} x$, where by convention we put $c=4$. The Schrödinger equation (3.1) is written

$$
\frac{\partial^{2} \psi}{\partial x^{2}}+\left(2 \operatorname{sech}^{2} x+\lambda\right) \psi=0
$$

To study equation (3.8), one poses

$$
\psi=A \operatorname{sech}^{\alpha} x . w(x)
$$

where $A$ is an arbitrary amplitude, $\alpha^{2}=-\lambda$ and $w$ satisfies the equation

$$
\frac{\partial^{2} w}{\partial x^{2}}-2 \alpha \tanh x \frac{\partial w}{\partial x}+\left(2+\alpha-\alpha^{2}\right) \operatorname{sech}^{2} x \cdot w=0 .
$$

By doing the substitution $u=\frac{1}{2}(1-\tanh x)$, the last equation comes down to a hypergeometric differential equation or Gaussian equation :

$$
u(1-u) \frac{\partial^{2} w}{\partial u^{2}}+(c-(a+b+1) u) \frac{\partial w}{\partial u}-a b w=0,
$$


where $a, b, c$ denote constants and are equal to $a=2+\alpha, b=-1+\alpha, c=1+\alpha$. This equation presents three regular singular points : $u=0, u=1, u=\infty$. The solution of this equation for $u=0$ is

$$
w \equiv F(a, b, c, u)=1+\frac{a b}{c} \cdot \frac{u}{1 !}+\frac{a(a+1) b(b+1)}{c(c+1)} \cdot \frac{u^{2}}{2 !}+\frac{a(a+1) \ldots(a+n-1) b(b+1) \ldots(b+n-1)}{c(c+1) \ldots(c+n-1)} \cdot \frac{u^{n}}{n !}+\cdots
$$

For $x \rightarrow \infty$ (i.e., when $u \rightarrow 0$ ), we have $w \rightarrow 1$. According to (3.9), we have $\psi=A 2^{\alpha}\left(e^{x}+e^{-x}\right)^{-\alpha} \cdot w(x)$ and this one tends to $A e^{2 \alpha} e^{-\alpha x}$ when $x \rightarrow \infty$. To represent a plane wave $A e^{i k x}$ going to $+\infty$, we will put $\alpha=-i k$. The asymptotic form of the wave function for $x \rightarrow-\infty(u \rightarrow 1)$ is obtained by transforming the hypergeometric function using the well-known functional relation

$$
F(a, b, c, u)=\frac{\Gamma(c) \Gamma(c-a-b)}{\Gamma(c-a) \Gamma(c-b)} F(a, b, a+b-c+1,1-u)+(1-u)^{c-a-b} \frac{\Gamma(c) \Gamma(a+b-c)}{\Gamma(a) \Gamma(b)} F(c-a, c-b, c-a-b+1,1-u),
$$

where $\Gamma(z)=\int_{0}^{\infty} e^{-t} e^{z-1} d t, \operatorname{Re} z>0$, is the Euler Gamma function. Taking into account (3.10) and the expression above, the relation (3.9) becomes

$$
\begin{aligned}
& \psi=A \operatorname{sech}^{\alpha} x {\left[\frac{\Gamma(c) \Gamma(c-a-b)}{\Gamma(c-a) \Gamma(c-b)}\left(1+\frac{a b}{a+b-c+1}(1-u)+\cdots\right)\right.} \\
&\left.+(1-u)^{c-a-b} \frac{\Gamma(c) \Gamma(a+b-c)}{\Gamma(a) \Gamma(b)}\left(1+\frac{(c-a)(c-b)}{c-a-b+1}(1-u)+\cdots\right)\right] .
\end{aligned}
$$

When $u \rightarrow 1(x \rightarrow-\infty)$, we have $(1-u)^{c-a-b} \rightarrow e^{-2 \alpha x}$ and since $\alpha=-i k$, then

$$
\psi \longrightarrow A e^{\alpha} \frac{\Gamma(c) \Gamma(a+b-c)}{\Gamma(a) \Gamma(b)}\left(e^{i k x}+\frac{\Gamma(c-a-b) \Gamma(a) \Gamma(b)}{\Gamma(c-a) \Gamma(c-b) \Gamma(a+b-c)}\right) .
$$

This expression combined with the fact that $\psi$ tends to $A e^{2 \alpha} e^{-\alpha}, x \rightarrow \infty$, give us the transmission coefficient $T$ and the reflection coefficient $R$,

$$
T=\frac{\Gamma(a) \Gamma(b)}{\Gamma(c) \Gamma(a+b-c)}, \quad R=\frac{\Gamma(c-a-b) \Gamma(a) \Gamma(b)}{\Gamma(c-a) \Gamma(c-b) \Gamma(a+b-c)} .
$$

We have $k_{1}=1, c(0)=\sqrt{2}, R(k, 0)=0$. For an individual soliton, equation (1.1) has a precise solution. It turns out that the soliton of amplitude $u_{0}$ has only one discrete level with eigenvalue $\lambda=\frac{u_{0}}{2}$, while the next level corresponds to the point $\lambda=0$ (with the respective eigenfunction $\psi=\tanh x$ ) and already belongs to the continuous spectrum. The Gelfand-Levitan equation (3.6) where $I(\mu, t)=c_{1}^{2}(t) e^{k_{1} \mu}=c_{1}^{2}(0) e^{-8 k_{1} t} e^{k_{1} t}=2 e^{-8 t+\mu}$ is written

$$
K(x, y ; t)+2 e^{-8 t+x+y}+2 e^{-8 t+y} \int_{-\infty}^{x} e^{z} K(x, z ; t) d z=0 .
$$

By putting $K(x, y, t)=f(x) e^{y}$, we obtain $f(x)+2 e^{-8 t+x}+e^{-8 t+2 x} f(x)=0$, hence, $f(x)=-2 \frac{e^{-x}}{1+e^{8 t-2 x}}$. Therefore, the solution (3.7) of the KdV equation in the case of a solitary wave is

$$
u(x, t)=2 \frac{d}{d x} K(x, x, t)=-\frac{2}{\cosh ^{2}(x-4 t)}=-2 \operatorname{sech}^{2}(x-4 t) .
$$

We will now look at the case of $N$-solitons through the procedure suggested by Gardner, Green, Kruskal, Miura [8] and to use the results of [15]. In order to solve the Gelfand-Levitan equation (3.6), where $R(k, t)=0$, one poses

$$
K(x, y)=\sum_{n=1}^{N} w_{n}(x, t) e^{k_{n} y}
$$

where $w_{n}$ are functions to be determined. By replacing this expression in the Gelfand-Levitan equation, we obtain the following system of linear algebraic equations for $w_{n}, n=1, \ldots, N$ :

$$
\left\{\begin{array}{l}
w_{1}(x, t)+c_{1}^{2}(t) e^{k_{1} x}+\sum_{m=1}^{N} c_{1}^{2}(t) \frac{e^{\left(k_{1}+k_{m}\right) x}}{k_{1}+k_{m}} w_{m}(x, t)=0, \\
\vdots \\
w_{N}(x, t)+c_{N}^{2}(t) e^{k_{N} x}+\sum_{m=1}^{N} c_{N}^{2}(t) \frac{e^{\left(k_{N}+k_{m}\right) x}}{k_{N}+k_{m}} w_{m}(x, t)=0 .
\end{array}\right.
$$


Define the following notations : $A=\left(c_{n}^{2}(t) e^{\left(k_{n}+k_{m}\right) x}\right), W=\left(w_{1} \cdots w_{N}\right)^{\top}, G=\left(c_{1}^{2}(t) e^{k_{1} x} \cdots c_{N}^{2}(t) e^{k_{N} x}\right)^{\top}$,

$$
P \equiv\left(P_{n m}\right)=\left(\delta_{n m}+c_{n}^{2}(t) \frac{e^{\left(k_{n}+k_{m}\right) x}}{k_{n}+k_{m}}\right)=I+A,
$$

where $I$ is the unit matrix. With these notations, the system above is written $P W=-G$, and it is easy to show that it has a unique solution. From equation (3.11), we draw $K(x, x)=h^{\top} w=-h^{\top} P^{-1} G, h \equiv\left(e^{k_{1} x} \cdots e^{k_{N} x}\right)^{\top}$. Or

$$
\frac{d}{d x} P_{n m}=c_{m}^{2} e^{k_{n} x} \cdot e^{k_{m} x}, \quad \operatorname{det} P=\sum_{n=1}^{N}\left(\delta_{n m}+c_{n}^{2}(t) \frac{e^{\left(k_{n}+k_{m}\right) x}}{k_{n}+k_{m}}\right) \alpha_{n m},
$$

and $P^{-1}=\frac{\alpha_{n m}}{\operatorname{det} P}$, where $\alpha_{n m}$ is the cofactor of $P$, so

$$
K(x, x)=-\sum_{n, m} \frac{\alpha_{n m}}{\operatorname{det} P} \frac{d}{d x} P_{n m}=-\frac{1}{\operatorname{det} P} \frac{d}{d x}(\operatorname{det} P)=-\frac{d}{d x} \ln \operatorname{det} P,
$$

and according to (3.7), we have $u=2 \frac{d}{d x} K(x, x)=-2 \frac{d^{2}}{d x^{2}} \ln \operatorname{det} P$. Therefore,

Theorem 3.2. The solution of the KdV equation is given by the function

$$
u=-2 \frac{d^{2}}{d x^{2}} \ln \operatorname{det} P
$$

where $P$ is defined by (3.12) and whose $c_{n}(t)=c_{n}(0) e^{-4 k_{n}^{3} t}$, with $k_{n}>0$ distinct.

The function obtained in the this theorem is negative for all $x$, continuous and behaves like the exponential when $|x| \rightarrow \infty$. To get an idea of the behavior of solitons and in particular their asymptotic behavior, suppose that $k_{1}<k_{2}<\ldots<k_{N-1}<k_{N}$. We will need the following result :

$$
\Delta \equiv\left|\begin{array}{cccc}
\frac{1}{a_{1}-b_{1}} & \frac{1}{a_{1}-b_{2}} & \cdots & \frac{1}{a_{1}-b_{n}} \\
\frac{1}{a_{2}-b_{1}} & \frac{1}{a_{2}-b_{2}} & \cdots & \frac{1}{a_{2}-b_{n}} \\
\vdots & & \ddots & \\
\frac{1}{a_{n}-b_{1}} & \frac{1}{a_{n}-b_{2}} & \cdots & \frac{1}{a_{n}-b_{n}}
\end{array}\right|=(-1)^{\frac{n(n-1)}{2}} \frac{\prod_{j<k}\left(a_{j}-a_{k}\right) \prod_{j<k}\left(b_{j}-b_{k}\right)}{\prod_{j, k}\left(a_{j}-b_{k}\right)} .
$$

Consider the following determinant :

$$
\Delta=\left|\begin{array}{ccc}
1+c_{1}^{2} b_{11} & c_{1}^{2} b_{12} & c_{1}^{2} b_{13} \\
c_{2}^{2} b_{21} & 1+c_{2}^{2} b_{22} & c_{2}^{2} b_{23} \\
c_{3}^{2} b_{31} & c_{3}^{2} b_{32} & 1+c_{3}^{2} b_{33}
\end{array}\right|
$$

where $c_{1} c_{2} c_{3} \neq 0$. If we divide the $1^{\text {st }}$ line by $c_{1}$, the $2^{\text {nd }}$ line by $c_{2}$ and the $3^{\text {nd }}$ line by $c_{3}$, we will have

$$
\Delta=c_{1} c_{2} c_{3}\left|\begin{array}{ccc}
\frac{1+c_{1}^{2} b_{11}}{c_{1}} & c_{1} b_{12} & c_{1} b_{13} \\
c_{2} b_{21} & \frac{1+c_{2}^{2} b_{22}}{c_{2}} & c_{2} b_{23} \\
c_{3} b_{31} & c_{3} b_{32} & \frac{1+c_{3}^{2} b_{33}}{c_{3}}
\end{array}\right|
$$

Multiply the $1^{\text {st }}$ column by $c_{1}$, the $2^{\text {nd }}$ column by $c_{2}$ and the $3^{\text {nd }}$ column by $c_{3}$, we get

$$
\Delta=\left|\begin{array}{ccc}
1+c_{1}^{2} b_{11} & c_{1} c_{2} b_{12} & c_{1} c_{3} b_{13} \\
c_{2} c_{1} b_{21} & 1+c_{2}^{2} b_{22} & c_{2} c_{3} b_{23} \\
c_{3} c_{1} b_{31} & c_{3} c_{2} b_{32} & 1+c_{3}^{2} b_{33}
\end{array}\right|
$$

So for the determinant of order $N$, just use the same procedure, i.e., by dividing the $j^{\text {nd }}$ row by $c_{j}$ and multiply the $j^{\text {nd }}$ column by $c_{j}$ 
Theorem 3.3. The explicit solution of $N$-solitons of the $K d V$ equation is given by

$$
u(x, t)= \begin{cases}-2 \sum_{n=1}^{N} k_{n}^{2} \operatorname{sech}^{2}\left(k_{n} \xi_{n}+\delta_{n}^{+}\right), & t \rightarrow+\infty \\ -2 \sum_{n=1}^{N} k_{n}^{2} \operatorname{sech}^{2}\left(k_{n} \xi_{n}+\delta_{n}^{-}\right), & t \rightarrow-\infty\end{cases}
$$

where the phase changes are given by

$$
\delta_{n}^{+} \equiv \frac{1}{2} \ln \frac{c_{n}^{2}}{2 k_{n}}\left(\prod_{j=1}^{n-1} \frac{k_{j}-k_{n}}{k_{j}+k_{n}}\right)^{2}, \quad \delta_{n}^{-} \equiv \frac{1}{2} \ln \frac{c_{n}^{2}}{2 k_{n}}\left(\prod_{j=n+1}^{N} \frac{k_{j}-k_{n}}{k_{j}+k_{n}}\right)^{2} .
$$

Proof. The determinant of the matrix $P$ is written explicitly in the form

$$
\operatorname{det} P=\left|\begin{array}{cccccc}
1+\frac{c_{1}^{2}(t)}{2 k_{1}} e^{2 k_{1} x} & \frac{c_{1}^{2}(t)}{k_{1}+k_{2}} e^{\left(k_{1}+k_{2}\right) x} & \ldots & \frac{c_{1}^{2}(t)}{k_{1}+k_{j}} e^{\left(k_{1}+k_{j}\right) x} & \ldots & \frac{c_{1}^{2}(t)}{k_{1}+k_{N}} e^{\left(k_{1}+k_{N}\right) x} \\
\frac{c_{2}^{2}(t)}{k_{2}+k_{1}} e^{\left(k_{2}+k_{1}\right) x} & 1+\frac{c_{2}^{2}(t)}{2 k_{2}} e^{2 k_{2} x} & & \frac{c_{2}^{2}(t)}{k_{2}+k_{j}} e^{\left(k_{2}+k_{j}\right) x} & \ldots & \frac{c_{2}^{2}(t)}{k_{2}+k_{N}} e^{\left(k_{2}+k_{N}\right) x} \\
\vdots & & & \ddots & & \\
\frac{c_{N}^{2}(t)}{k_{N}+k_{1}} e^{\left(k_{N}+k_{1}\right) x} & \frac{c_{N}^{2}(t)}{k_{N}+k_{2}} e^{\left(k_{N}+k_{2}\right) x} & \ldots & \frac{c_{N}^{2}(t)}{k_{N}+k_{j}} e^{\left(k_{N}+k_{j}\right) x} & & 1+\frac{c_{N}^{2}(t)}{2 k_{N}} e^{2 k_{N} x}
\end{array}\right|
$$

Applying the previous remark to the determinant $\operatorname{det} P$ above, we obtain

$$
\operatorname{det} P=\left|\begin{array}{cccc}
1+\frac{c_{1}^{2}(t)}{2 k_{1}} e^{2 k_{1} x} & \frac{c_{1}(t) c_{2}(t)}{k_{1}+k_{2}} e^{\left(k_{1}+k_{2}\right) x} & \ldots & \frac{c_{1}(t) c_{N}(t)}{k_{1}+k_{N}} e^{\left(k_{1}+k_{N}\right) x} \\
\frac{c_{2}(t) c_{1}(t)}{k_{2}+k_{1}} e^{\left(k_{2}+k_{1}\right) x} & 1+\frac{c_{2}^{2}(t)}{2 k_{2}} e^{2 k_{2} x} & \ldots & \frac{c_{2}(t) c_{N}(t)}{k_{2}+k_{N}} e^{\left(k_{2}+k_{N}\right) x} \\
\vdots & & \ddots & \\
\frac{c_{N}(t) c_{1}(t)}{k_{N}+k_{1}} e^{\left(k_{N}+k_{1}\right) x} & \frac{c_{N}(t) c_{2}(t)}{k_{N}+k_{2}} e^{\left(k_{N}+k_{2}\right) x} & \ldots & 1+\frac{c_{N}^{2}(t)}{2 k_{N}} e^{2 k_{N} x}
\end{array}\right|
$$

Since $c_{j}(t)=c_{j}(0) e^{-4 k_{j}^{3} t}$, then

$$
\operatorname{det} P=\left|\begin{array}{cccc}
1+\frac{c_{1}^{2}(0)}{2 k_{1}} e^{2 k_{1} \xi_{1}} & \frac{c_{1}(0) c_{2}(0)}{k_{1}+k_{2}} e^{k_{1} \xi_{1}+k_{2} \xi_{2}} & \ldots & \frac{c_{1}(0) c_{N}(0)}{k_{1}+k_{N}} e^{k_{1} \xi_{1}+k_{N} \xi_{n}} \\
\frac{c_{2}(0) c_{1}(0)}{k_{2}+k_{1}} e^{k_{2} \xi_{2}+k_{1} \xi_{1}} & 1+\frac{c_{2}^{2}(0)}{2 k_{2}} e^{2 k_{2} \xi_{2}} & \ldots & \frac{c_{2}(0) c_{N}(0)}{k_{2}+k_{N}} e^{k_{2} \xi_{2}+k_{N} \xi_{N}} \\
\vdots & & \ddots & \\
\frac{c_{N}(0) c_{1}(0)}{k_{N}+k_{1}} e^{k_{N} \xi_{N}+k_{1} \xi_{1}} & \frac{c_{N}(0) c_{2}(0)}{k_{N}+k_{2}} e^{k_{N} \xi_{N}+k_{2} \xi_{2}} & \ldots & 1+\frac{c_{N}^{2}(0)}{2 k_{N}} e^{2 k_{N} \xi_{N}}
\end{array}\right|
$$

where $\xi_{n} \equiv x-4 k_{j}^{2} t, 1 \leq j \leq N$. To get an idea of the behavior of solitons and in particular their asymptotic behavior, suppose that $k_{1}<k_{2}<\ldots<k_{N-1}<k_{N}$. For $t \gg 0$ let's write $\xi_{j}$ in the form $\xi_{j} \equiv \xi_{n}-\varepsilon_{j n} t, 1 \leq j \leq N$ with $\varepsilon_{j n} \equiv 4 k_{j}^{2}-4 k_{n}^{2}$ and $c_{j}(0) \equiv c_{j}$. Note that $\varepsilon_{j n}<0$ if $1 \leq j<n, \varepsilon_{n n}=0, \varepsilon_{j n}>0$ if $n<j \leq N$, and $\varepsilon_{j n}=-\varepsilon_{n j}$. We have also $\varepsilon_{n m}>\varepsilon_{(n-1) m}>\ldots>\varepsilon_{(m+1) n}>0$ if $n>m$, and $\varepsilon_{n m}<\varepsilon_{n(m-1)}<\ldots<\varepsilon_{n(m+1)}<0$ if $n<m$. Replace these expressions in the determinant above and approximate the elements of the diagonal (for $j<n$ ):

$$
1+\frac{c_{j}^{2}}{2 k_{j}} e^{2 k_{j}\left(\xi_{n}-\varepsilon_{j n} t\right)} \cong \frac{c_{j}^{2}}{2 k_{j}} e^{2 k_{j}\left(\xi_{n}-\varepsilon_{j n} t\right)}, \quad j<n, \quad t \rightarrow \infty
$$

(we can do it because for $j<n$, we have $\varepsilon_{j n}<0$ and $1+e^{x} \cong e^{x}$ for $x \rightarrow \infty$ ). Then, we put in factor the following common expressions : $e^{2 k_{1}\left(\xi_{n}-\varepsilon_{1 n} t\right)}, e^{2 k_{2}\left(\xi_{n}-\varepsilon_{2 n} t\right)}, \ldots, e^{2 k_{n-1}\left(\xi_{n}-\varepsilon_{(n-1) n} t\right)}$. By turning $t$ to infinity, we have (since $\varepsilon_{j n}>0$ for $\left.n \leq j \leq N\right)$ the 
following situation :

$$
\operatorname{det} P=C\left|\begin{array}{cccccccc}
\frac{c_{1}^{2}}{2 k_{1}} & \frac{c_{1} c_{2}}{k_{1}+k_{2}} & \ldots & \frac{c_{1} c_{n-1}}{k_{1}+k_{n-1}} & \frac{c_{1} c_{n}}{k_{1}+k_{n}} e^{k_{n} \xi_{n}} & 0 & \ldots & 0 \\
\frac{c_{2} c_{1}}{k_{2}+k_{1}} & \frac{c_{2}^{2}}{2 k_{2}} & \ldots & \frac{c_{2} c_{n-1}}{k_{2}+k_{n-1}} & \frac{c_{2} c_{n}}{k_{2}+k_{n}} e^{k_{n} \xi_{n}} & 0 & \ldots & 0 \\
\vdots & & \ddots & & \vdots & \vdots & \ldots & \vdots \\
\frac{c_{n-1} c_{1}}{k_{n-1}+k_{1}} & \frac{c_{n-1} c_{2}}{k_{n-1}+k_{2}} & \ldots & \frac{c_{n-1}^{2}}{2 k_{n-1}} & \frac{c_{n-1} c_{n}}{k_{n-1}+k_{n}} e^{k_{n} \xi_{n}} & 0 & \ldots & 0 \\
\frac{c_{n} c_{1}}{k_{n}+k_{1}} e^{k_{n} \xi_{n}} & \frac{c_{n} c_{2}}{k_{n}+k_{2}} e^{k_{n} \xi_{n}} & \ldots & \frac{c_{n} c_{n-1}}{k_{n}+k_{n-1}} e^{k_{n} \xi_{n}} & 1+\frac{c_{n}^{2}}{2 k_{n}} e^{2 k_{n} \xi_{n}} & 0 & \ldots & 0 \\
0 & 0 & \ldots & 0 & 0 & 1 & \ldots & 0 \\
\vdots & \vdots & \vdots & \vdots & \vdots & \vdots & \ddots & \vdots \\
0 & 0 & \ldots & 0 & 0 & 0 & \ldots & 1
\end{array}\right|
$$

where $C \equiv \prod_{j=1}^{n-1} e^{2 k_{j}\left(\xi_{n}-\varepsilon_{j n}\right)}$. Obviously, we have

$$
\operatorname{det} P=C\left|\begin{array}{ccccc}
\frac{c_{1}^{2}}{2 k_{1}} & \frac{c_{1} c_{2}}{k_{1}+k_{2}} & \ldots & \frac{c_{1} c_{n-1}}{k_{1}+k_{n-1}} & \frac{c_{1} c_{n}}{k_{1}+k_{n}} e^{k_{n} \xi_{n}} \\
\frac{c_{2} c_{1}}{k_{2}+k_{1}} & \frac{c_{2}^{2}}{2 k_{2}} & \ldots & \frac{c_{2} c_{n-1}}{k_{2}+k_{n-1}} & \frac{c_{2} c_{n}}{k_{2}+k_{n}} e^{k_{n} \xi_{n}} \\
\vdots & & \ddots & & \vdots \\
\frac{c_{n-1} c_{1}}{k_{n-1}+k_{1}} & \frac{c_{n-1} c_{2}}{k_{n-1}+k_{2}} & \ldots & \frac{c_{n-1}^{2}}{2 k_{n-1}} & \frac{c_{n-1} c_{n}}{k_{n-1}+k_{n}} e^{k_{n} \xi_{n}} \\
\frac{c_{n} c_{1}}{k_{n}+k_{1}} e^{k_{n} \xi_{n}} & \frac{c_{n} c_{2}}{k_{n}+k_{2}} e^{k_{n} \xi_{n}} & \ldots & \frac{c_{n} c_{n-1}}{k_{n}+k_{n-1}} e^{k_{n} \xi_{n}} & 1+\frac{c_{n}^{2}}{2 k_{n}} e^{2 k_{n} \xi_{n}}
\end{array}\right| .
$$

This determinant is still written in the form

$$
\operatorname{det} P=C \prod_{l=1}^{n-1} c_{l}^{2}\left|\begin{array}{cccc}
\frac{1}{2 k_{1}} & \frac{1}{k_{1}+k_{2}} & \cdots & \frac{1}{k_{1}+k_{n-1}} \\
\frac{1}{k_{2}+k_{1}} & \frac{1}{2 k_{2}} & \cdots & \frac{1}{k_{2}+k_{n-1}} \\
\vdots & & \ddots & \vdots \\
\frac{1}{k_{n-1}+k_{1}} & \frac{1}{k_{n-1}+k_{2}} & \cdots & \frac{1}{2 k_{n-1}}
\end{array}\right|+C \prod_{l=1}^{n} c_{l}^{2}\left|\begin{array}{ccccc}
\frac{1}{2 k_{1}} & \frac{1}{k_{1}+k_{2}} & \cdots & \frac{1}{k_{1}+k_{n-1}} & \frac{1}{k_{1}+k_{n}} \\
\frac{1}{k_{2}+k_{1}} & \frac{1}{2 k_{2}} & \cdots & \frac{1}{k_{2}+k_{n-1}} & \frac{1}{k_{2}+k_{n}} \\
\vdots & & \ddots & & \vdots \\
\frac{1}{k_{n-1}+k_{1}} & \frac{1}{k_{n-1}+k_{2}} & \cdots & \frac{1}{2 k_{n-1}} & \frac{1}{k_{n-1}+k_{n}} \\
\frac{1}{k_{n}+k_{1}} & \frac{1}{k_{n}+k_{2}} & \cdots & \frac{1}{k_{n}+k_{n-1}} & \frac{1}{2 k_{n}}
\end{array}\right|,
$$

for $n \geq 2$, while for $n=1$, it equals to $1+\frac{c_{1}^{2}}{2 k_{1}} e^{2 k_{1} \xi_{1}}$. Using the previous lemma, we get for $t \gg 0$,

$$
\operatorname{det} P=\prod_{i=1}^{n-1} e^{2 k_{i}\left(\xi_{n}-\varepsilon_{i n} t\right)}\left(\prod_{j=1}^{n-1} c_{j}^{2} \frac{\left(\prod_{i<j}\left(k_{i}-k_{j}\right)\right)^{2}}{\prod_{i, j}\left(k_{i}+k_{j}\right)}+\prod_{j=1}^{n-1} c_{j}^{2} \frac{\left(\prod_{i<j}\left(k_{i}-k_{j}\right)\right)^{2}}{\prod_{i, j}\left(k_{i}+k_{j}\right)} e^{2 k_{n} \xi_{n}}\right) .
$$

By replacing this expression in the solution obtained in the last theorem, we obtain the explicit solution of $N$-solitons :

$$
u(x, t)=-2 \sum_{n=1}^{N} k_{n}^{2} \operatorname{sech}^{2}\left(k_{n} \xi_{n}+\frac{1}{2} \ln \frac{c_{n}^{2}}{2 k_{n}}\left(\prod_{j=1}^{n-1} \frac{k_{j}-k_{n}}{k_{j}+k_{n}}\right)^{2}\right), \quad t \rightarrow+\infty .
$$

Similarly, it is shown that for $t \ll 0$

$$
u(x, t)=-2 \sum_{n=1}^{N} k_{n}^{2} \operatorname{sech}^{2}\left(k_{n} \xi_{n}+\frac{1}{2} \ln \frac{c_{n}^{2}}{2 k_{n}}\left(\prod_{j=n+1}^{N} \frac{k_{j}-k_{n}}{k_{j}+k_{n}}\right)^{2}\right), \quad t \rightarrow-\infty .
$$

This completes the demonstration.

This result can be interpreted as follows: for example for $t \rightarrow \infty$, we have

$$
\lim _{t \rightarrow \infty} u(x, t)=\lim _{t \rightarrow \infty} u(x-c t)=\left\{\begin{aligned}
-2 k_{n}^{2} \operatorname{sech}^{2}\left(k_{n}\left(x-4 k_{n}^{2} t\right)+\delta_{n}^{+}\right) & \text {if } c=4 k_{n}^{2} \\
0 & \text { if } c \neq 4 k_{n}^{2}
\end{aligned}\right.
$$


This is the form of a solitary wave of amplitude $2 k_{n}^{2}$, propagating on the right with a constant velocity equal to $4 k_{n}^{2}$. The solution of the KdV equation actually splits into $N$-solitons at the limit for $|t| \rightarrow \infty$. This indicates that each soliton preserves its shape after collisions. These are analyzed by the phase changes $\delta_{n}^{+}$and $\delta_{n}^{-}$. The relative phase change is determined by

$$
\delta_{n}^{+}-\delta_{n}^{-}=\frac{1}{2} \ln \frac{c_{n}^{2}}{2 k_{n}}\left(\prod_{j=1}^{n-1} \frac{k_{j}-k_{n}}{k_{j}+k_{n}}\right)^{2}-\frac{1}{2} \ln \frac{c_{n}^{2}}{2 k_{n}}\left(\prod_{j=n+1}^{N} \frac{k_{j}-k_{n}}{k_{j}+k_{n}}\right)^{2}=\sum_{j=1}^{n-1} \ln \frac{k_{j}-k_{n}}{k_{j}+k_{n}}-\sum_{j=n+1}^{N} \ln \frac{k_{j}-k_{n}}{k_{j}+k_{n}},
$$

and it is expressed in terms of $k_{j}(1 \leq j \leq N)$. Since $k_{j}$ are invariant with respect to time, then the $\delta_{n}^{+}-\delta_{n}^{-}$are also invariant. Recall that we assumed $k_{1}<k_{2}<\ldots<k_{N}$, then

$$
\delta_{1}^{+}-\delta_{1}^{-}=-\sum_{j=2}^{N} \ln \frac{k_{j}-k_{1}}{k_{j}+k_{1}}>0, \quad \delta_{N}^{+}-\delta_{N}^{-}=\sum_{j=1}^{N-1} \ln \frac{k_{N}-k_{1}}{k_{N}+k_{1}}<0 .
$$

In addition, it is easy to show that $\sum_{n=1}^{N} \delta_{n}^{+}=\sum_{n=1}^{N} \delta_{n}^{-}$.

We could not finish this section without indicating some results related to the KdV equation. The KdV equation (1.1) is written in the form

$$
\frac{\partial u}{\partial t}=\frac{\partial}{\partial x}\left(3 u^{2}-\frac{\partial^{2} u}{\partial x^{2}}\right)=\frac{\partial}{\partial x} \frac{\delta H}{\delta u}
$$

where

$$
H=\int_{-\infty}^{\infty} P_{3} d x=\int_{-\infty}^{\infty}\left(u^{3}+\frac{1}{2}\left(\frac{\partial u}{\partial x}\right)^{2}\right) d x
$$

is the first integral (Hamiltonian) obtained previously and $\frac{\delta H}{\delta u(x)}$ denotes the gradient (Fréchet derivative) of the function $H$. This equation forms an infinite dimensional Hamiltonian system, completely integrable and the Hamiltonian structure is defined by the Poisson bracket : $\{F, H\}=\int \frac{\delta F}{\delta u(x)} \frac{\partial}{\partial x} \frac{\delta H}{\delta u(x)} d x$. We check that the latter satisfies the Jacobi identity. We will discuss further (in the following sections) the problem of studying the $\mathrm{KdV}$ equation via symplectic structures on operator algebra, the relation with the KP hierarchy [16], the Sato theory [17] $\tau$ functions and the work of Jimbo-Miwa-Kashiwara [18, 19]. The study of the periodic problem for the KdV equation allowed some authors to discover an interesting class of completely integrable systems. The obtained solutions are endowed with remarkable properties : they define functions $u(x)$ for which equation (2.1) with periodic coefficients has a finite number of zones of parametric resonance on the axis $\lambda$. The spectrum of the Schrödinger operator is invariant by the Hamiltonian flow defined by the KdV equation. And as we have already pointed out, this spectrum provides an infinity first integrals or invariants. The isospectral sets related to invariant manifolds defined by putting these invariants equal to generic constants are compact, connected, and infinite-dimensional tori. Each of these isospectral sets is isomorphic to the real part of a Jacobi variety associated with a hyperelliptic curve of finite or infinite genus. The periods of this torus can be expressed using hyperelliptic integrals; in short, the explicit linearization of the flow of the $\mathrm{KdV}$ equation is made on this Jacobian variety using the Abel application, the Jacobi inversion problem and the theta functions. For other interesting integrable systems that will not be discussed here, the solutions blow up after a finite time as Laurent series depending on many parameters (see for example [20, 21]).

\section{Pseudo-differential operators}

Let $L$ be a pseudo-differential operator with holomorphic coefficients. The set of these operators form a Lie algebra that we note $\mathscr{A}$. The algebra $\mathscr{A}$ decomposes in two sub-algebras $\mathscr{A}_{+}$and $\mathscr{A}_{-}: \mathscr{A}=\mathscr{A}_{+} \oplus \mathscr{A}_{-}$, where $\mathscr{A}_{+}$is the algebra of differential operators of the form $\zeta=\sum_{k \geq 0} u_{k}(x) \partial^{k}$, finite sum, $\partial=\frac{\partial}{\partial x}$, and $\mathscr{A}_{-}$is the algebra of strictly pseudo-differential operators of the form

$$
\eta=\sum_{k>0} u_{-k}(x) \partial^{-k}=\partial^{-1} v_{0}+\partial^{-2} v_{1}+\cdots, \quad \partial=\frac{\partial}{\partial x},
$$


The algebra $\mathscr{A}$ is an associative algebra for the product of two pseudo-differential operators $L$ and $L^{\prime}$,

$$
L . L^{\prime}=\sum_{k=0}^{\infty} \frac{1}{k !}: \partial_{\partial}^{k}(L) . \partial_{x}^{k}\left(L^{\prime}\right):
$$

where $\partial=\frac{\partial}{\partial x}$ and the symbol :: denotes the normal order, i.e., it means that the derivatives always appear on the right independently of the commutation relations.

For $m, n \in \mathbb{N}^{*}$, we have for all functions $u, v$,

$$
u \partial^{m} \cdot v \partial^{n}=\sum_{k=0}^{m} \frac{m !}{k !(m-k) !} u v^{(k)} \partial^{m+n-k}=\sum_{k=0}^{m} \frac{1}{k !}: \partial_{\partial}^{k}\left(u \partial^{m}\right) \cdot \partial_{x}^{k}\left(v \partial^{n}\right):
$$

and

$$
\partial^{-1} u=u \partial^{-1}-u^{\prime} \partial^{-2}+u^{\prime \prime} \partial^{-3}+\cdots=\sum_{k=0}^{\infty}: \partial_{\partial}^{k}\left(\partial^{-1}\right) \cdot \partial_{x}^{k}(u):
$$

where $\partial^{-1}$ is a formal inverse of $\partial$, i.e., $\partial^{-1} . \partial=\partial . \partial^{-1}=1$.

We define a coupling between $\mathscr{A}_{+}$and $\mathscr{A}_{-}$as follows : Let $\operatorname{Res}(\zeta \eta)$ be the coefficient of $\partial^{-1}$ in $\zeta \eta$. We have

$$
\langle\zeta, \eta\rangle=\left\langle\sum_{k \geq 0} u_{k} \partial^{k}, \sum_{k>0} u_{-k} \partial^{-k}\right\rangle=\left\langle u_{0} \partial^{0}+u_{1} \partial^{1}+\cdots, \partial^{-1} v_{0}+\partial^{-2} v_{1}+\cdots\right\rangle
$$

i.e., $\langle\zeta, \eta\rangle=\int_{-\infty}^{\infty} \operatorname{Res}(\zeta \eta) d x=\int_{-\infty}^{\infty} \sum_{k \geq 0} u_{k} v_{k} d x$. Therefore, the Volterra group $\left(I+\mathscr{A}_{-}\right)$acts on $\mathscr{A}_{-}$by the adjoint action and on $\mathscr{A}_{+}$by the coadjoint action. Let $\zeta \in \mathscr{A}_{+}$and $\eta_{k} \in \mathscr{A}_{-}$. We obtain from [22],

$$
\begin{aligned}
\left\langle a d_{\eta_{1}}^{*}(\zeta), \eta_{2}\right\rangle=\left\langle\zeta, a d_{\eta_{1}}\left(\eta_{2}\right)\right\rangle & =\left\langle\zeta,\left[\eta_{1}, \eta_{2}\right]\right\rangle \\
& =\int\left(\partial^{-1}-\text { term of }\left(\zeta \eta_{1} \eta_{2}-\zeta \eta_{2} \eta_{1}\right)\right) d x \\
& =\int\left(\partial^{-1}-\text { term of }\left(\zeta \eta_{1}-\eta_{1} \zeta\right)_{+} \eta_{2}\right) d x \\
& =\left\langle\left[\zeta, \eta_{1}\right]_{+}, \eta_{2}\right\rangle .
\end{aligned}
$$

So the set $\mathscr{O}_{\mathscr{A}_{+}}^{*}(L)$ of the differential operators of the form

$$
L=\partial^{N}+\sum_{k=0}^{N-2} u_{k}(x) \partial^{k}, \quad N \text { fixed }
$$

is a coadjoint orbit in $\mathscr{A}_{+}$.

Let $f$ be a function of class $\mathscr{C}^{\infty}$ in $x$ and dependent on a finite number of derivatives $u_{k}^{(l)}$ of the coefficients $u_{k}$ of $L$. Let

$$
\nabla H(L)=\sum_{k=0}^{N-1} \partial^{-k-1} \sum_{l}(-1)^{l}\left(\frac{d}{d x}\right)^{l} \frac{\partial f}{\partial p_{k}^{(l)}}=\sum_{k=0}^{N-1} \partial^{-k-1} \frac{\delta H}{\delta u_{k}}
$$

be the gradient of the functional, $H(L)=\int_{-\infty}^{\infty} f\left(x, \ldots, u_{k}^{(l)}, \ldots\right) d x$ defined on $\mathscr{A}_{+}$and such that :

$$
d H=\int_{-\infty}^{\infty} \frac{\delta H}{\delta u_{k}} d u_{k}=\left\langle\sum_{k=0}^{N} d u_{k} \cdot \partial^{k}, \nabla H\right\rangle=\langle d L, \nabla H\rangle
$$

where $d L=\sum_{k=0}^{N} d u_{k} \cdot \partial^{k}$. We recall that the scalar product between two pseudo-differential operators $L$ and $L^{\prime}$ is defined by $\left.\left\langle L, L^{\prime}\right\rangle=-\int_{-\infty}^{\infty}\left(L L^{\prime}\right)_{-} d x=\int_{-\infty}^{\infty}\left(L^{\prime} L\right)_{-} d x\right)$. According to the Adler-Kostant-Symes [20, 22, 23, 24, 25, 26, 27, 28, 29], the Hamiltonian vector fields on the coadjoint orbit $\mathscr{O}_{\mathscr{A}_{+}}^{*}$, define commutative flows and are given by

$$
\frac{d L}{d t}=a d_{\nabla H(L)}^{*}(L)=[L, \nabla H(L)]_{+},
$$


where $H(L)$ is the Hamiltonian on $\mathscr{A}_{+}$. The operator $L$ does not contain the coefficient $u_{N-1}$. Since the vector field (4.4) applied to the operator $L(4.3)$ imposes the condition $\operatorname{Res}[L, \nabla H(L)]=0$, we can replace the gradient $\frac{\delta H}{\delta p_{N-1}}$ by any expression satisfying this condition. A first Poisson bracket is given by

$$
\{H, F\}_{1}=\langle L,[\nabla F, \nabla H]\rangle=\int \operatorname{Res}\left(\nabla H[L, \nabla F]_{+}\right) d x=\int \operatorname{Res}(\nabla H[L, \nabla F]) d x=\int \operatorname{Res}([\nabla H, L] \nabla F) d x .
$$

Consider the Hamiltonians $H_{k+N}=\frac{N}{k+N} \int\left(\operatorname{Rés} L^{\frac{k+N}{N}}\right) d x, k \in \mathbb{N}^{*}$. We have $\nabla H_{k+N}^{(L)}=\left(L^{\frac{k}{N}}\right)_{-}$, and the vector fields (4.4) applied to these Hamiltonians, provide the integrable equations; $N$-reduction of Gel'fand Dickey equations of KP hierarchy (see below for definition) :

$$
\frac{d L}{d t}=\left[L, \nabla H_{k+N}(L)\right]_{+}=-\left[\left(L^{\frac{k}{N}}\right)_{-}, L\right]_{+}=\left[\left(L^{\frac{k}{N}}\right)_{+}, L\right] .
$$

Note that since $\left[\left(L^{\frac{k}{N}}\right)_{+}, L\right]_{+}=\left[L^{\frac{k}{N}}-\left(L^{\frac{k}{N}}\right)_{-}, L\right]_{+}=-\left[\left(L^{\frac{k}{N}}\right)_{-}, L\right] \in \mathscr{A}^{-}$, then equations (4.6) determine an infinite number of commutative vector fields (see below) on $\mathscr{A}^{+}+\mathscr{A}^{-}$.

We will now study $[22,30,31,32,33]$ the existence of a second symplectic structure. Let $\widetilde{L}=L+z$ where $L$ is a differential operator of order $n$. We have

$$
\frac{d L}{d t}=(\widetilde{L} \nabla H)_{+} \widetilde{L}-\widetilde{L}(\nabla H \widetilde{L})_{+} .
$$

Note that (4.7) is a Hamiltonian vector field generalizing (4.4). Indeed, let $J: \mathscr{A}_{-} / \mathscr{A}_{-\infty, N-1} \longrightarrow \mathscr{D}_{0, N-1}$, be the function defined by

$$
J(\zeta)=(\widetilde{L} \zeta)_{+} \widetilde{L}-\widetilde{L}(\zeta \widetilde{L})_{+}=-(\widetilde{L} \zeta)_{-} \widetilde{L}+\widetilde{L}(\zeta \widetilde{L})_{-}, \quad \zeta \in \mathscr{A}_{-} / \mathscr{A}_{-\infty, N-1}
$$

Hence, $\frac{d L}{d t}=\partial_{J(\zeta)}(L) \equiv(\widetilde{L} \zeta)_{+} \widetilde{L}-\widetilde{L}(\zeta \widetilde{L})_{+}$, which shows that it is indeed a vector field on the differential operators $L$ of order $n$. Similarly, we have $\frac{d L}{d t}=-(\widetilde{L} \nabla H)_{-} \widetilde{L}+\widetilde{L}(\nabla H \widetilde{L})_{-}$, and the same conclusion remains valid. We also have the relation $\frac{d L}{d t}=(L \nabla H)_{+} L-L(\nabla H L)_{+}+z[\nabla H, L]_{+}$, which shows that this vector field is an interpolation between (4.4) for $z=\infty$ and a new vector field for $z=0$. Consider the 2-differential form

$$
\omega\left(\partial_{J(\zeta)}, \partial_{J(\eta)}\right)=\langle J(\zeta), \eta\rangle=\int \operatorname{Res}(J(\zeta) \eta) d x
$$

This form is closed $(d \omega=0)$ and is antisymmetric $\langle J(\zeta), \eta\rangle=-\langle\zeta, J(\eta)\rangle$, and furthermore $\left[\partial_{J(\zeta)}, \partial_{J(\eta)}\right]=\partial_{J(\xi)}$, where

$$
\xi=\left(-\zeta(\widetilde{L} \eta)_{+}+(\zeta \widetilde{L})_{-} \eta\right)_{-}\left(-\eta(\widetilde{L} \zeta)_{+}+(\eta \widetilde{L})_{-} \zeta\right)_{-}+\partial_{J(\zeta)} \eta-\partial_{J(\eta)} \zeta
$$

The functional algebra on the operator space of the form (4.3) for this symplectic form is the so called $\mathscr{W}$ algebra.

Theorem 4.1. The Hamiltonians $H_{k}, H_{k+N}, H_{k+2 N}, \ldots$, defined in (4.6) are all in involution for the bracket (4.5).

Proof. Indeed, let $J=J_{1}$ if $z=\infty$ and $J=J_{2}$ if $z=0$, where the Poisson brackets $\{., .\}_{1},\{., .\}_{2}$ are given by $\left\{H_{j}, H_{k}\right\}_{1}=$ $\int \operatorname{Res}\left(\nabla H_{j} J_{1}\left(\nabla H_{k}\right)\right)$ and

$$
\left\{H_{j}, H_{k}\right\}_{2}=\left\langle\nabla H, J_{2}(\nabla F)\right\rangle=\int \operatorname{Res}\left(\nabla H\left((L \nabla F)_{+} L-L(\nabla F L)_{+}\right)\right) d x=\int \operatorname{Res}\left(L \nabla H(L \nabla F)_{+}-\nabla H L(\nabla F L)_{+}\right) d x .
$$

We deduce from the relation

$$
\left(L\left(L^{\frac{r}{n}-1}\right)_{-}\right)_{+} L-L\left(\left(L^{\frac{r}{n}-1}\right)_{-} L\right)_{+}+\left[\left(L^{\frac{r}{n}}\right)_{-}, L\right]_{+}=0
$$


the expression $\left\{H_{j}, H_{k}\right\}_{1}=\int \operatorname{Res}\left(\nabla H_{j} J_{2} \nabla H_{k-N}\right)$. Since the form $\omega$ is anti-symmetric, we have

$$
\begin{aligned}
& \left\{H_{j}, H_{k}\right\}_{1}=-\int \operatorname{Res}\left(\nabla H_{k-N} J_{2}\left(\nabla H_{j}\right)\right)=-\int \operatorname{Res}\left(\nabla H_{k-N} J_{1}\left(\nabla H_{j+N}\right)\right), \\
& \left\{H_{j}, H_{k}\right\}_{1}=-\int \operatorname{Res}\left(\nabla H_{j+N} J_{1}\left(\nabla H_{k-N}\right)\right), \\
& \left\{H_{j}, H_{k}\right\}_{1}=\left\{H_{j+N}, H_{k-N}\right\}_{1}=\left\{H_{j}, H_{k}\right\}_{1}=\left\{H_{j+\alpha N}, H_{k-\alpha N}\right\}_{1},
\end{aligned}
$$

for $\alpha$ large enough with $J_{1}\left(\nabla H_{k-\alpha N}\right)=0$, i.e., $H_{k-\alpha N}$ is trivial for $\alpha$ large enough and we get $\left\{H_{j}, H_{k}\right\}_{1}=0$, so $H_{j}, H_{k}$ are in involution.

\section{KdV equation, Heisenberg and Virasoro algebras}

Theorem 5.1. a) The operator $L=\partial^{2}+q$, corresponding to the case $N=2$ with $q \equiv u_{0}$, is related to the KdV equation and the Poisson bracket is provided in this case by $\{q(x), q(y)\}_{1}=\frac{d}{d x} \delta(x-y)$.

b) By replacing in a), $q(x)$ by the Fourier series

$$
q(x)=\alpha \sum_{n=-\infty}^{\infty} e^{-i n x} \varphi_{n}+\beta, \quad-i \alpha^{-2}=1,
$$

where $\left(\varphi_{k}\right)_{k \in \mathbb{Z}}$ are new coordinates (Fourier coefficients), one obtains the Heisenberg algebra and the Poisson bracket is provided by $\left\{\varphi_{n}, \varphi_{m}\right\}_{1}=n \delta_{m+n, 0}$.

c) In the case $N=2$ one obtains the Virasoro algebra and its structure is given by

$$
\left\{\varphi_{m}, \varphi_{n}\right\}_{2}=(m-n) \varphi_{m+n}+\frac{c}{12}\left(m^{3}-m\right) \delta_{m+n, 0} .
$$

Proof. a) Indeed, since

$$
\nabla H(L)=\partial^{-1} \frac{\delta H}{\delta q}+\partial^{-2} \frac{1}{2}\left(\frac{\delta H}{\delta q}\right)^{\prime}
$$

then the vector fields applied to the Hamiltonian $H=\int\left(q^{3}-\frac{1}{2} q^{\prime 2}\right) d x$, provide the KdV equation

$$
\frac{d q}{d t}=\frac{d L}{d t}=\frac{1}{2}[L, \nabla H]_{+}=\frac{d}{d x} \frac{\delta H}{\delta q}=\frac{d q}{d t}=\frac{\partial^{3} q}{\partial x^{3}}+6 q \frac{\partial q}{\partial x} .
$$

The Poisson bracket is in this case is $\{H, F\}_{1}=\int \frac{\delta H}{\delta q} \frac{d}{d x} \frac{\delta F}{\delta q}$, and therefore, $\{q(x), q(y)\}_{1}=\frac{d}{d x} \delta(x-y)$.

b) Let $\mathscr{M}$ be the set of matrices $\left(a_{k l}\right),(k, l \in \mathbb{Z})$, with complex coefficients and

$$
\mathscr{N}=\left\{\left(a_{k l}\right) \in \mathscr{M}: \text { there is at least } r \text { such that } a_{k l}=0 \text { for }|k-l|>r\right\},
$$

the $\mathbb{C}$-algebra, i.e., the set of infinite matrices with support in a band around the diagonal. The product of two matrices belonging respectively to $\mathscr{N}$ and $\mathscr{M}$ is defined in the usual way. Note that $\mathscr{N}$ is a Lie algebra and $\mathscr{M}$ is a $\mathscr{N}$-module. Their extensions $\widetilde{\mathscr{N}}$ and $\widetilde{\mathscr{M}}$ are defined by

$$
0 \longrightarrow \mathbb{C} c \longrightarrow \widetilde{\mathscr{N}} \longrightarrow \mathscr{N} \longrightarrow 0, \quad 0 \longrightarrow \mathbb{C} c \longrightarrow \widetilde{\mathscr{M}} \longrightarrow \mathscr{M} \longrightarrow 0
$$

with $\widetilde{\mathscr{N}}=\mathscr{N} \oplus \mathbb{C} c, \widetilde{\mathscr{M}}=\mathscr{M} \oplus \mathbb{C} c$, where $c$ is a central element, i.e., we have $[c, A]=[c, B]=0, \forall A \in \widetilde{\mathscr{N}}, \forall B \in \widetilde{\mathscr{M}}$. We notice $e_{i, j}=\left(\delta_{k i} . \delta_{l j}\right)_{k l}$ the elementary matrices, i.e., the matrices whose coefficients are all zero except the one of the line $i$ and the column $j$ which is equal to 1 . Since a Jacobi matrix has no trace, then we consider the matrix $A[J, B]$ where $A \in \mathscr{N}, B \in \mathscr{M}$ and $J$ is the matrix defined by $J=\sum_{i \in \mathbb{Z}} \varepsilon(i) e_{i, i}$, where $\varepsilon(i)=+1$ if $i<0$ and -1 if $i \geq 0$. The elements of the matrix $A[J, B]$ are null except for a finite number, so it is a finite matrix and we define the cocycle of $A \in \mathscr{N}$ and $B \in \mathscr{M}$ using the formula

$$
\rho(A, B)=\frac{1}{2} \operatorname{Tr}(A[J, B])=\frac{1}{2} \sum_{i, j}(\varepsilon(i)-\varepsilon(j)) a_{i j} b_{j i} .
$$


Therefore, the bracket [,] of $A \in \mathscr{N}$ and $B \in \mathscr{M}$ is defined by

$$
\widetilde{[A, B]}=[A+\alpha c, B+\beta c]=[A, B]+\rho(A, B) c .
$$

We note that the $\widetilde{\mathscr{N}}$ algebra is a non-trivial central extension of $N$ while the subalgebra $\widetilde{\mathscr{M}_{f}}=\mathscr{M}_{f} \oplus \mathbb{C} c$, is a trivial central extension of

$$
\mathscr{M}_{f}=\left\{\left(a_{i j}\right) \in \mathscr{M}:(i, j) \longmapsto\left(a_{i j}\right) \text { with finished support }\right\}
$$

Let's put $E_{i}=\sum_{n \in \mathbb{Z}} e_{n, n+i}$, where $e_{i, i}=\left(\delta_{k i} . \delta_{i j}\right)_{k l}$ are the elementary matrices defined above. The subspace $E=\bigoplus_{i \in \mathbb{Z}} \mathbb{C} E_{i}$, is a commutative subalgebra of $\mathscr{N}$. The subalgebra of $\mathscr{N}$ defined by setting $\widetilde{E}=E \oplus \mathbb{C} c$ is called Heisenberg subalgebra. We have

$$
\widetilde{\left[E_{i}, E_{j}\right]}=i \delta_{i,-j} c \text {. }
$$

We now reconsider the previous example and replace $q(x)$ with the Fourier series $(5.1)$. Let $H$ be a functional of $q$. Its Fréchet derivative in terms of the coordinates $\varphi_{k}$ is written

$$
\frac{\delta H}{\delta q}=\sum_{k=-\infty}^{\infty} \frac{\delta H}{\delta \varphi_{k}} \cdot \frac{\partial \varphi_{k}}{\partial q}=\alpha^{-1} \sum_{k=-\infty}^{\infty} \frac{\delta H}{\delta \varphi_{k}} e^{i k x}
$$

We substitute (5.3) and (5.1) in equation (5.2) and we specify the Fourier coefficients; we get the relation $\alpha \frac{\partial \varphi_{n}}{\partial t}=-i \alpha^{-1} n \frac{\partial H}{\partial \varphi_{n}}$. Moreover, since the symplectic structure is given by the matrix of the Poisson brackets, we also have

$$
\frac{\partial \varphi_{n}}{\partial t}=\sum_{m=-\infty}^{\infty}\left\{\varphi_{n}, \varphi_{m}\right\}_{1} \frac{\partial H}{\partial \varphi_{m}}
$$

Therefore, $\left\{\varphi_{n}, \varphi_{m}\right\}_{1}=-i \alpha^{-2} n \delta_{m+n, 0}$. By putting $-i \alpha^{-2}=1$, we obtain the Heisenberg algebra (where $\{$,$\} plays the role$ here of the bracket $\widetilde{[,]}(5.3)$ above).

c) Let $\operatorname{Diff}\left(S^{1}\right)$ be the group of diffeomorphisms of the unit circle : $S^{1}=\{z \in \mathbb{C}:|z|=1\}$. Let

$$
F=\left\{f(z) \frac{d}{d z}: f(z) \in \mathbb{C}\left[z, \frac{1}{z}\right]\right\},
$$

be the set of vector fields (Laurent's polynomials). Note that $F$ can be seen as the tangent space Diff $\left(S^{1}\right)$ at its unit point, so $F$ is a Lie algebra with respect to the bracket [,]. By setting $\varphi_{m}=-z^{m+1} \frac{d}{d z}$, we obtain

$$
\left[\varphi_{m}, \varphi_{n}\right]=\left((n+1) z^{m+n+1}-(m+1)^{m+n+1}\right) \frac{d}{d z}=-(m+n) z^{m+n+1} \frac{d}{d z}
$$

i.e., $\left[\varphi_{m}, \varphi_{n}\right]=(m-n) \varphi_{m+n}$. We show that $H^{2}(F, \mathbb{C}) \cong \mathbb{C}$ and $\rho\left(\varphi_{m}, \varphi_{n}\right)=\frac{1}{12}\left(m^{3}-m\right) \delta_{m,-n}$. The vector space $F \oplus \mathbb{C} c$ is called Virasoro algebra, it is a central extension of the algebra of complex vector fields on the circle. The bracket is given by the formula

$$
\left[\varphi_{m}, \varphi_{n}\right]=(m-n) \varphi_{m+n}+\frac{c}{12}\left(m^{3}-m\right) \delta_{m,-n} .
$$

Let us now consider the example of the $\mathrm{KdV}$ equation. We have $N=2$ and

$$
\frac{d q}{d t}=\frac{d L}{d t}=(L \nabla H)_{+}-L(\nabla H L)_{+}=\left(\partial^{3}+2(\partial q+q \partial)\right) \frac{\delta H}{\delta q} .
$$

The (Poisson) bracket is written in this case

$$
\{H, F\}_{2}=\int \frac{\delta H}{\delta q}\left(\partial^{3}+2(\partial q+q \partial)\right) \frac{\delta F}{\delta q},
$$


and we have $\{q(x), q(y)\}_{2}=\left(\partial^{3}+2(\partial q+q \partial)\right) \delta(x-y)$. By reasoning as before (while taking into account the Fréchet derivative (5.4)), we obtain

$$
\alpha \frac{\partial \varphi_{m}}{\partial t}=i \sum_{n}(n-m) \varphi_{m+n} \frac{\delta H}{\delta \varphi_{n}}+\frac{i}{2 \alpha}\left(m^{3}-4 \beta m\right) \frac{\delta H}{\delta \varphi_{-m}},
$$

where $\left(\varphi_{k}\right)_{k \in \mathbb{Z}}$ are the Fourier coefficients of $q$. By setting $4 \beta=1, \alpha=\frac{6 i}{c}$ and taking into account the Fourier series (5.1), we obtain

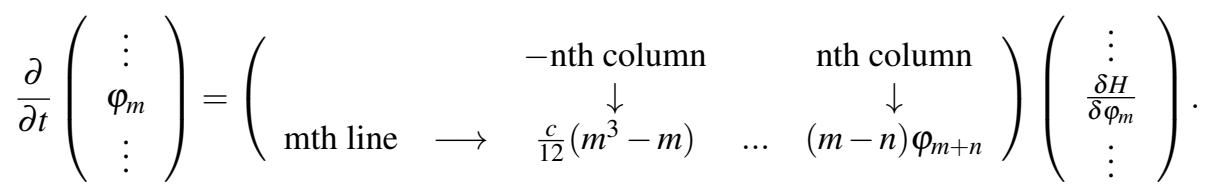

Consequently, we have $\left\{\varphi_{m}, \varphi_{n}\right\}_{2}=(m-n) \varphi_{m+n}+\frac{c}{12}\left(m^{3}-m\right) \delta_{m+n, 0}$, i.e., the Virasoro structure [19] (where $\{,\}_{2}$ plays the role here of the bracket [,] (5.5) above). This establishes the theorem.

$$
\begin{aligned}
& \text { For } N=3, u \equiv u_{2}, v \equiv u_{3}, L=\partial^{3}+u \partial+v, \text { and } L^{\frac{2}{3}}=\partial^{3}+\frac{2}{3} u, \text { the flow (5.2) takes the form } \\
& \qquad \frac{\partial u}{\partial t_{2}}=-\frac{\partial^{2} u}{\partial x^{2}}+2 \frac{\partial v}{\partial x}, \quad \frac{\partial v}{\partial t_{2}}=\frac{\partial^{2} v}{\partial x^{2}}-\frac{2}{3} \frac{\partial^{3} u}{\partial x^{3}}-\frac{2}{3} u \frac{\partial u}{\partial x}
\end{aligned}
$$

Eliminating $v$ from these equations yields the Boussinesq equation

$$
3\left(\frac{\partial u}{\partial t_{2}}\right)^{2}+\frac{\partial^{2}}{\partial x^{2}}\left(\frac{\partial^{2} u}{\partial x^{2}}+2 u^{2}\right)=0
$$

\section{KP hierarchy and vertex operators}

Consider the pseudo-differential operator of infinite order

$$
L=\partial+u_{1} \partial^{-1}+u_{2} \partial^{-2}+\ldots, \quad \partial \equiv \frac{\partial}{\partial x}
$$

where $u_{1}, u_{2}, \ldots$ are functions of class $\mathscr{C}^{\infty}$ depending on an infinity of independent variables $x \equiv t_{1}, t_{2}, \ldots$. The compound operator $L^{n}$ is calculated according to the rules (4.1) and (4.2). We obtain

$$
L^{n}=\partial^{n}+p_{n, 2} \partial^{n-2}+\ldots+p_{n, n}+p_{n, n+1} \partial^{-1}+\ldots=\partial^{n}+\sum_{j=2}^{n} p_{n, j} \partial^{n-j}+\sum_{j=1}^{\infty} p_{n, n+j} \partial^{-j},
$$

where $p_{n, j}$ are polynomials in $u_{j}$ and their derivatives in relation to $x$. The differential part $L_{+}^{n}$ of $L^{n}$ being equal to $L_{+}^{n}=$ $\partial^{n}+\sum_{j=2}^{n} p_{n, j} \partial^{n-j}$, we have

$$
L_{+}^{1}=\partial, \quad L_{+}^{2}=\partial^{2}+2 u_{2}, \quad L_{+}^{3}=\partial^{3}+3 u_{2} \partial+3\left(u_{3}+\partial u_{2}\right), \ldots
$$

The dependency between the functions $u_{1}, u_{2}, \ldots$ and the variables $x=t_{1}, t_{2}, \ldots$ is provided by the following system of partial differential equations :

$$
\frac{\partial L}{\partial t_{n}}=\left[L_{+}^{n}, L\right], \quad n \in \mathbb{N}^{*}
$$

The set of these equations is called Kadomtsev-Petviashvili hierarchy (abbreviated KP hierarchy). It is a hierarchy of isospectral deformations of the pseudo-differential operator (6.1). We prove (see [34]) the following result : 
Theorem 6.1. There is an equivalence between (6.3) and the equations

$$
\frac{\partial}{\partial t_{n}} L_{+}^{m}-\frac{\partial}{\partial t_{m}} L_{+}^{n}=\left[L_{+}^{n}, L_{+}^{m}\right]
$$

as well as their dual forms

$$
\frac{\partial}{\partial t_{n}} L_{-}^{m}-\frac{\partial}{\partial t_{m}} L_{-}^{n}=-\left[L_{-}^{n}, L_{-}^{m}\right]
$$

where $L_{-}^{n}=L^{n}-L_{+}^{n}$. Equations (6.3) determine an infinite number of commutative vector fields on algebra $\mathscr{A}^{=} \mathscr{A}_{+} \oplus \mathscr{A}_{-}$.

Proof. Note that since $L^{n}=L_{+}^{n}+L_{-}^{n}$, then $\frac{\partial L}{\partial t_{n}}=\left[L_{+}^{n}, L\right]=-\left[L_{-}^{n}, L\right] \in \mathscr{A}_{-}$. Equation (6.3) defines an infinite number of vector fields on $\mathscr{A}$. Since $\frac{\partial}{\partial t_{n}}$ and $\left[L_{+}^{n},.\right]$ are derivations, then

$$
\begin{aligned}
\frac{\partial L^{m}}{\partial t_{n}} & =\left[L_{+}^{n}, L_{+}^{m}\right]+\left[L_{+}^{n}, L_{-}^{m}\right], \\
& =-\left[L_{-}^{n}, L_{+}^{m}\right]-\left[L_{-}^{n}, L_{-}^{m}\right], \\
& =\frac{1}{2}\left(\left[L_{+}^{n}, L_{+}^{m}\right]-\left[L_{-}^{n}, L_{+}^{m}\right]\right)+\frac{1}{2}\left(-\left[L_{-}^{n}, L_{-}^{m}\right]+\left[L_{+}^{n}, L_{-}^{m}\right]\right), \\
& =\frac{1}{2}\left(\left[L_{+}^{n}, L_{+}^{m}\right]-\left[L_{-}^{n}, L_{-}^{m}\right]\right)+\frac{1}{2}\left(\left[L_{+}^{m}, L_{-}^{n}\right]-\left[L_{-}^{m}, L_{+}^{n}\right]\right) .
\end{aligned}
$$

Similarly, we have (just swap $n$ and $m$ )

$$
\frac{\partial L^{n}}{\partial t_{m}}=\frac{1}{2}\left(\left[L_{+}^{m}, L_{+}^{n}\right]-\left[L_{-}^{m}, L_{-}^{n}\right]\right)+\frac{1}{2}\left(\left[L_{+}^{n}, L_{-}^{m}\right]-\left[L_{-}^{n}, L_{+}^{m}\right]\right) .
$$

Hence, $\frac{\partial L^{m}}{\partial t_{n}}-\frac{\partial L^{n}}{\partial t_{m}}=\left[L_{+}^{n}, L_{+}^{m}\right]-\left[L_{-}^{n}, L_{-}^{m}\right]$. Or

$$
\frac{\partial L^{m}}{\partial t_{n}}-\frac{\partial L^{n}}{\partial t_{m}}=\frac{\partial}{\partial t_{n}} L_{+}^{m}+\frac{\partial}{\partial t_{n}} L_{-}^{m}-\frac{\partial}{\partial t_{m}} L_{+}^{n}-\frac{\partial}{\partial t_{m}} L_{-}^{n}=\frac{\partial}{\partial t_{n}} L_{+}^{m}-\frac{\partial}{\partial t_{m}} L_{+}^{n}+\frac{\partial}{\partial t_{n}} L_{-}^{m}-\frac{\partial}{\partial t_{m}} L_{-}^{n},
$$

then

$$
\frac{\partial}{\partial t_{n}} L_{+}^{m}-\frac{\partial}{\partial t_{m}} L_{+}^{n}-\left[L_{+}^{n}, L_{+}^{m}\right]=-\frac{\partial}{\partial t_{n}} L_{-}^{m}+\frac{\partial}{\partial t_{m}} L_{-}^{n}-\left[L_{-}^{n}, L_{-}^{m}\right] .
$$

Since the expression on the left belongs to $\mathscr{A}_{+}$and the one on the right belongs to $\mathscr{A}_{-}$, then the result comes from the decomposition $\mathscr{A}=\mathscr{A}_{+} \oplus \mathscr{A}_{-}$since obviously $\mathscr{A}_{+} \cap \mathscr{A}_{-}=\emptyset$. To show that the vector fields defined by these equations commute, we put $X(L)=\left[L_{+}^{m}, L\right]$ and $Y(L)=\left[L_{+}^{n}, L\right]$. Hence,

$$
\begin{aligned}
{[X, Y](L) } & =(X Y-Y X)(L), \\
& =X\left(\left[L_{+}^{n}, L\right]\right)-Y\left(\left[L_{+}^{m}, L\right]\right), \\
& =\left[X\left(L_{+}^{n}\right)-Y\left(L_{+}^{m}\right), L\right]+\left[L_{+}^{n}, X(L)\right]-\left[L_{+}^{m}, Y(L)\right], \\
& =\left[X\left(L_{+}^{n}\right)-Y\left(L_{+}^{m}\right), L\right]+\left[L_{+}^{n},\left[L_{+}^{m}, L\right]\right]-\left[L_{+}^{m},\left[L_{+}^{n}, L\right]\right], \\
& =\left[X\left(L_{+}^{n}\right)-Y\left(L_{+}^{m}\right)-\left[L_{+}^{m}, L_{+}^{n}\right], L\right],
\end{aligned}
$$

according to Jacobi's identity and taking into account (6.4), we deduce that the vector fields in question commute.

By specifying the quantifiers of $\partial^{k}$ in (6.4), one obtains an infinity of nonlinear partial differential equations [5] forming the Kadomtsev-Petviashvili hierarchy. These equations connect infinitely many functions $u_{j}$ to infinitely many variables $t_{j}$. For example, for $m=2, n=3$, relations (6.4) and (6.2) determine two expressions based on $u_{2}$ and $u_{3}$. After eliminating $u_{3}$, we immediately obtain the Kadomtsev-Petviashvili equation (KP equation) :

$$
3 \frac{\partial^{2} u_{2}}{\partial t_{2}^{2}}-\frac{\partial}{\partial t_{1}}\left(4 \frac{\partial u_{2}}{\partial t_{3}}-12 u_{2} \frac{\partial u_{2}}{\partial t_{1}}-\frac{\partial^{3} u_{2}}{\partial t_{1}^{3}}\right)=0 .
$$


We can obtain particular solutions of this equation by solving the equations :

$$
\frac{\partial u_{2}}{\partial t_{2}}=0, \quad 4 \frac{\partial u_{2}}{\partial t_{3}}-12 u_{2} \frac{\partial u_{2}}{\partial t_{1}}-\frac{\partial^{3} u_{2}}{\partial t_{1}^{3}}=0 .
$$

The second equation is precisely the KdV equation. The KP equation is therefore a generalization of the KdV equation, to which it is reduced when $\frac{\partial u_{2}}{\partial t_{2}}=0$.

Equations (6.3) and (6.4) (see also (6.5)) imply the existence of the following pseudo-differential operator of degree 0 (wave operator) $W \in \mathscr{I}+\mathscr{A}_{-}$:

$$
W=1+w_{1}(t) \partial^{-1}+w_{2}(t) \partial^{-2}+\cdots
$$

with $t=\left(t_{1}, t_{2}, \ldots\right) \in \mathbb{C}^{\infty}$. The inverse $W^{-1}$ of $W$ is also a pseudo-differential operator of the form

$$
W^{-1}=1+v_{1}(t) \partial^{-1}+v_{2}(t) \partial^{-2}+\cdots,
$$

and can be calculated term by term. Indeed, by definition, we have $W W^{-1}=1$. So, using the fact that

$$
\partial^{m} u=\sum_{k=0}^{\infty} \frac{m !}{k !(m-k) !}\left(\partial^{k} u \partial^{u}\right) \partial^{m-k} u, \quad \partial^{m} \partial^{n}=\partial^{m+n},
$$

as well as the formulas described above, we specify the quantifiers of $\partial^{-1}, \partial^{-2}, \ldots$ in the equation $W W^{-1}=1$ and we determine relations between $w_{m}$ et $v_{m}$. We obtain finally for $W^{-1}$ the following expression :

$$
W^{-1}=1-w_{1} \partial^{-1}+\left(-w_{2}+w_{1}^{2}\right) \partial^{-2}+\left(w_{3}+2 w_{1} w_{2}-w_{1} \partial w_{1}-w_{1}^{3}\right) \partial^{-3}+\cdots
$$

In terms of $W$, the operator $L(6.1)$ can be written in the form

$$
L=W \cdot \partial \cdot W^{-1} .
$$

According to (6.1) and ((6.7), we deduce the relations :

$$
u_{2}=\partial w_{2}, \quad u_{3}=-\partial w_{2}-w_{1} \partial w_{1}, \quad u_{4}=-\partial w_{3}+w_{1} \partial w_{2}+\left(\partial w_{1}\right) w_{2}-w_{1}^{2} \partial w_{1}-\left(\partial w_{1}\right)^{2} .
$$

We have the following result :

Theorem 6.2. Equations (6.3) or what amounts to the same equations (6.4)) are equivalent to the existence of the wave operator $W$ (6.7) such that the system of differential equations

$$
\begin{aligned}
& L W=W \partial, \\
& \frac{\partial W}{\partial t_{n}}=-L_{-}^{n} W,
\end{aligned}
$$

has a solution (which can be inductively obtained).

Theorem 6.3. a) Let $\xi(t, z)=\sum_{j=1}^{\infty} t_{j} z^{j}, z \in \mathbb{C}$ be the phase function with $\partial^{m} \xi(t, z)=z^{m}$ and $\partial^{m} e^{\xi(t, z)}=z^{m} e^{\xi(t, z)}$. There is an equivalence between (6.6), (6.10) and the following problem : there is a wave function $\psi$ (Baker-Akhiezer function)

$$
\Psi(t, z)=\left(1+w_{1}(t) z^{-1}+w_{2}(t) z^{-2}+\cdots\right) e^{\xi(t, z)}=W e^{\xi(t, z)}, \quad z \in \mathbb{C}
$$

where $W$ is identified as (6.7) and such that :

$$
L \Psi=z \Psi, \quad \frac{\partial \Psi}{\partial t_{n}}=L_{+}^{n} \Psi .
$$

b) Introduce the conjugation $\partial^{*}=-\partial$ and let

$$
L^{*}=1+(-\partial)^{-1} u_{1}+(-\partial)^{-2} u_{2}+\cdots, \quad W^{*}=1+(-\partial)^{-1} w_{1}+(-\partial)^{-2} w_{2}+\cdots
$$

be the adjoints of $L$ and $W$ such that : $L^{*}=-\left(W^{*}\right)^{-1} . \partial . W^{*}$. The adjoint wave function

$$
\Psi^{*}(t, z)=\left(W^{*}(t, \partial)\right)^{-1} e^{-\xi(t, z)},
$$

satisfies the following relations : $L^{*} \Psi^{*}=z \Psi^{*}, \frac{\partial \Psi^{*}}{\partial t_{n}}=-\left(L_{+}^{n}\right)^{*} \Psi^{*}$. 
Proof. a) Indeed, we have from (6.11),

$$
\begin{aligned}
\frac{\partial \Psi}{\partial t_{n}} & =\frac{\partial W}{\partial t_{n}} e^{\xi(t, z)}+W z^{n} e^{\xi(t, z)} \\
& =-L_{-}^{n} W e^{\xi(t, z)}+z^{n} W e^{\xi(t, z)}, \quad \text { according to }(6.8) \\
& =-L_{-}^{n} \Psi+z^{n} \Psi, \quad \text { according to (6.9) } \\
& =-L_{-}^{n} \Psi+L^{n} \Psi, \quad \text { according to (6.10) } \\
& =L_{+}^{n} \Psi .
\end{aligned}
$$

In other words, $\Psi$ satisfies (6.11) and (6.12) is equivalent to the fact that $W$ satisfies (6.7) and (6.10).

b) Just reason as in the proof of a).

Therefore, the knowledge of $\Psi$ implies the knowledge of $W$ and also of $W^{*}$ and $L$. Define the following residues : $\operatorname{Res}_{z} \sum a_{k} z^{k}=a_{-1}, \operatorname{Res}_{\partial} \sum a_{k} \partial^{k}=a_{-1}$ and consider the following result [33],

Theorem 6.4. Let $P$ and $Q$ be two pseudo-differential operators. So

$$
\underset{z}{\operatorname{Res}}\left(\left(P e^{x z}\right) \cdot\left(Q e^{-x z}\right)\right)=\underset{\partial}{\operatorname{Res} P Q^{*}}
$$

where $Q^{*}$ is the adjoint of $Q$.

Proof. Indeed, we have

$$
\operatorname{Res}_{z}\left(\left(P e^{x z}\right) \cdot\left(Q e^{-x z}\right)\right)=\operatorname{Res}_{z}\left(\sum p_{k} z^{k} \sum q_{l}(-z)^{l}\right)=\sum_{k+l=-1}(-1)^{l} p_{k} q_{l},
$$

and

$$
\operatorname{Res}_{\partial} P Q^{*}=\operatorname{Res}_{\partial} \sum_{k l} p_{k} \partial^{k}(-\partial)^{l} q_{l}=\sum_{k+l=-1}(-1)^{l} p_{k} q_{l},
$$

hence the result.

Moreover, we have [33, 34] :

$$
\begin{aligned}
\operatorname{Res}_{z}\left(\partial^{k} \Psi\right) . \Psi^{*} & =\operatorname{Res}_{z}\left(\partial^{k} W e^{\xi(t, z)}\right)\left(W^{*}\right)^{-1} e^{-\xi(t, z)}, \\
& =\operatorname{Res}_{z}\left(\partial^{k} W e^{x z}\right)\left(W^{*}\right)^{-1} e^{-x z}, \quad x \equiv t-1, \\
& =\operatorname{Res}_{\partial} \partial^{k} W \cdot W^{-1}, \\
& =\operatorname{Res}_{\partial} \partial^{k}, \\
& =0 .
\end{aligned}
$$

This bilinear identity can be written in the following symbolic form :

$$
\operatorname{Res}_{z=\infty}\left(\Psi(t, z) \cdot \Psi^{*}\left(t^{\prime}, z\right)\right)=0
$$

for all $t$ and $t^{\prime}$. Therefore, we have

Theorem 6.5. $\Psi(t, z)$ is a wave function for the KP hierarchy if and only if the residue identity is satisfied:

$$
\underset{z=\infty}{\operatorname{Res}}\left(\Psi(t, z) \cdot \Psi^{*}\left(t^{\prime}, z\right)\right)=0 \quad \forall t, t^{\prime}
$$

or what amounts to the same if and only if

$$
\frac{1}{2 \pi \sqrt{-1}} \int_{\gamma} \Psi(t, z) \cdot \Psi^{*}\left(t^{\prime}, z\right) d z=0
$$

with $\gamma$ a closed path around $z=\infty$ (such that $\left.: \int_{\gamma} \frac{d z}{2 \pi \sqrt{-1}}=1\right)$. 
Recall that a $\tau(t)$ function is defined by the Fay differential identity (see next theorem) :

$$
\left\{\tau\left(t-\left[y_{1}\right]\right), \tau\left(t-\left[y_{2}\right]\right)\right\}+\left(y_{1}^{-1}-y_{2}^{-1}\right)\left(\tau\left(t-\left[y_{1}\right]\right) \tau\left(t-\left[y_{2}\right]\right)-\tau(t) \tau\left(t-\left[y_{1}\right]-\left[y_{2}\right]\right)\right)=0,
$$

où $y_{1}, y_{2} \in \mathbb{C}^{*}$ and $\{u, v\}$ is the Wronskian $u^{\prime} v-u v^{\prime}$.

Theorem 6.6. Let's put $[s]=\left(s, \frac{s^{2}}{2}, \frac{s^{3}}{3}, \ldots\right)$. The $\tau$ function satisfies the following identities :

(i) Fay identity :

$$
\begin{aligned}
\mathscr{F}\left(t, y_{0}, y_{1}, y_{2}, y_{3}\right) \equiv & \left(y_{0}-y_{1}\right)\left(y_{2}-y_{3}\right) \tau\left(t+\left[y_{0}\right]+\left[y_{1}\right]\right) \tau\left(t+\left[y_{2}\right]+\left[y_{3}\right]\right) \\
& +\left(y_{0}-y_{2}\right)\left(y_{3}-y_{1}\right) \tau\left(t+\left[y_{0}\right]+\left[y_{2}\right]\right) \tau\left(t+\left[y_{2}\right]+\left[y_{1}\right]\right) \\
& +\left(y_{0}-y_{3}\right)\left(y_{1}-y_{2}\right) \tau\left(t+\left[y_{0}\right]+\left[y_{3}\right]\right) \tau\left(t+\left[y_{1}\right]+\left[y_{2}\right]\right) \\
= & 0 .
\end{aligned}
$$

(ii) Fay differential identity :

$$
\left\{\tau\left(t-\left[y_{1}\right]\right), \tau\left(t-\left[y_{2}\right]\right)\right\}+\left(y_{1}^{-1}-y_{2}^{-1}\right)\left(\tau\left(t-\left[y_{1}\right]\right) \tau\left(t-\left[y_{2}\right]\right)-\tau(t) \tau\left(t-\left[y_{1}\right]-\left[y_{2}\right]\right)\right)=0
$$

where $y_{1}, y_{2} \in \mathbb{C}^{*}$ and $\{u, v\}$ is the Wronskian $u^{\prime} v-u v^{\prime}$. This identity can still be written in the form

$$
\partial^{-1} \psi(t, \lambda) \psi^{*}(t, \mu)=\frac{1}{\mu-\lambda} \frac{\tau\left(t-\left[\lambda^{-1}\right]+\left[\mu^{-1}\right]\right)}{\tau(t)} e^{\sum_{j=1}^{\infty} t_{j}\left(\mu^{j}-\lambda^{j}\right)} .
$$

The following equation $\tau=X(t, \lambda, \mu) \tau$, determines a vector field on the infinite dimension manifold of the $\tau$ functions where $X(t, \lambda, \mu)$ is the vertex operator (of Date-Jimbo-Kashiwara-Miwa) for the KP equation.

Proof. According to Sato theory $[35,36]$, the functions $\Psi$ and $\Psi^{*}$ can be expressed in terms of a tau function as follows :

$$
\begin{aligned}
& \Psi(t, z)=W e^{\xi(t, z)}=\frac{\tau\left(t-\left[z^{-1}\right]\right)}{\tau(t)} e^{\xi(t, z)}, \\
& \Psi^{*}(t, z)=\left(W^{*}\right)^{-1} e^{-\xi(t, z)}=\frac{\tau\left(t+\left[z^{-1}\right]\right)}{\tau(t)} e^{-\xi(t, z)} .
\end{aligned}
$$

By replacing these expressions in the residue formula (6.13) or (6.14), we obtain a bilinear relation for the $\tau$ functions. Indeed, the equation (6.14) is written

$$
\int_{\gamma} e^{\xi\left(t-t^{\prime}, z\right)} \tau\left(t-\left[z^{-1}\right]\right) \tau\left(t^{\prime}-\left[z^{-1}\right]\right) d z=0
$$

'Using the following change $: t \leftarrow t+s$ and $t^{\prime} \leftarrow t+s$, we obtain

$$
\int_{\gamma} e^{\xi(-2 s, z)} \tau\left(t-s-\left[z^{-1}\right]\right) \tau\left(t+s+\left[z^{-1}\right]\right) d z=0 .
$$

Using again the transformation

$$
s \leftarrow t+\frac{1}{2}\left(\left[y_{0}\right]+\left[y_{1}\right]+\left[y_{2}\right]+\left[y_{3}\right]\right), \quad t \leftarrow \frac{1}{2}\left(\left[y_{0}\right]-\left[y_{1}\right]-\left[y_{2}\right]-\left[y_{3}\right]\right),
$$

and taking into account that $e^{\sum_{1}^{\infty}\left(a b^{-1}\right)^{j} \cdot j^{-1}}=1-a b^{-1}$, we obtain via the residue theorem

$$
\begin{aligned}
0 & =\int_{\gamma} \frac{1-z y_{0}}{\prod_{j=1}^{3}\left(1-z y_{j}\right)} \tau\left(t-s-\left[z^{-1}\right]\right) \tau\left(t+s+\left[z^{-1}\right]\right) d z, \\
& =2 \pi \sqrt{-1} \sum_{y_{1}^{-1}, y_{2}^{-1}, y_{3}^{-1}} \operatorname{Res}_{\prod_{j=1}^{3}\left(1-z y_{j}\right)}\left(\frac{1-z y_{0}}{2 \pi \sqrt{-1}}\left(t-s-\left[z^{-1}\right]\right) \tau\left(t+s+\left[z^{-1}\right]\right)\right), \\
& =\frac{\left.2 \pi y_{0}, y_{1}, y_{2}, y_{3}\right),}{\left(y_{1}-y_{2}\right)\left(y_{2}-y_{3}\right)\left(y_{3}-y_{1}\right)} \mathscr{F}\left(t, y^{2}\right)
\end{aligned}
$$


where

$$
\begin{aligned}
\mathscr{F}\left(t, y_{0}, y_{1}, y_{2}, y_{3}\right) \equiv & \left(y_{0}-y_{1}\right)\left(y_{2}-y_{3}\right) \tau\left(t+\left[y_{0}\right]+\left[y_{1}\right]\right) \tau\left(t+\left[y_{2}\right]+\left[y_{3}\right]\right) \\
& +\left(y_{0}-y_{2}\right)\left(y_{3}-y_{1}\right) \tau\left(t+\left[y_{0}\right]+\left[y_{2}\right]\right) \tau\left(t+\left[y_{2}\right]+\left[y_{1}\right]\right) \\
& +\left(y_{0}-y_{3}\right)\left(y_{1}-y_{2}\right) \tau\left(t+\left[y_{0}\right]+\left[y_{3}\right]\right) \tau\left(t+\left[y_{1}\right]+\left[y_{2}\right]\right) .
\end{aligned}
$$

The relation $\mathscr{F}\left(t, y_{0}, y_{1}, y_{2}, y_{3}\right)=0$ is the Fay identity. By making the transformation in the expression $\left.\left(y_{1} y_{2}\right)^{-1} \frac{\partial \mathscr{F}}{\partial y_{0}}\right|_{y_{0}=y_{3}=0}$ and replacing $t$ by $t-\left[y_{1}\right]-\left[y_{2}\right]$, we obtain the Fay differential identity which allows to define the $\tau$ functions :

$$
\left\{\tau\left(t-\left[y_{1}\right]\right), \tau\left(t-\left[y_{2}\right]\right)\right\}+\left(y_{1}^{-1}-y_{2}^{-1}\right)\left(\tau\left(t-\left[y_{1}\right]\right) \tau\left(t-\left[y_{2}\right]\right)-\tau(t) \tau\left(t-\left[y_{1}\right]-\left[y_{2}\right]\right)\right)=0,
$$

where $y_{1}, y_{2} \in \mathbb{C}^{*}$ and $\{u, v\}$ is the Wronskian $u^{\prime} v-u v^{\prime}$. Consider the Fay differential identity above and replace $t$ with $t+\left[y_{1}\right]$. We obtain

$$
\left\{\tau(t), \tau\left(t+\left[y_{1}\right]-\left[y_{2}\right]\right)\right\}+\left(y_{1}^{-1}-y_{2}^{-1}\right)\left(\tau(t) \tau\left(t+\left[y_{1}\right]-\left[y_{2}\right]\right)-\tau(t) \tau\left(t-\left[y_{2}\right]\right)\right)=0 .
$$

By putting $\lambda=y_{1}^{-1}, \mu=y_{2}^{-1}$, we obtain after having multiplied the expression obtained by $\frac{1}{\tau(t)} e^{\Sigma_{1}^{\infty} t_{j}\left(\mu^{j}-\lambda^{j}\right)}$, the following formula :

$$
\frac{\tau\left(t+\left[\lambda^{-1}\right]\right)}{\tau(t)} e^{-\sum t_{j} \lambda^{j}} \frac{\tau\left(t-\left[\mu^{-1}\right]\right)}{\tau(t)} e^{\sum t_{j} \mu^{j}}=\frac{1}{\mu-\lambda} \frac{\partial}{\partial x}\left(e^{\sum t_{j}\left(\mu^{j}-\lambda^{j}\right)} \frac{\tau\left(t+\left[\lambda^{-1}\right]-\left[\mu^{-1}\right]\right)}{\tau(t)}\right) .
$$

Let

$$
X(t, \lambda, \mu)=\frac{1}{\mu-\lambda} e^{\sum_{1}^{\infty} t_{j}\left(\mu^{j}-\lambda^{j}\right)} e^{\sum_{1}^{\infty} j^{-1}\left(\lambda^{-j}-\mu^{-j}\right) \frac{\partial}{\partial t_{j}}}, \quad \lambda \neq \mu
$$

be the vertex operator (of Date-Jimbo-Kashiwara-Miwa) for the KP equation, then $X(t, \lambda, \mu) \tau$ et $\tau+X(t, \lambda, \mu) \tau$ are also $\tau$ functions. Therefore, $\tau=X(t, \lambda, \mu) \tau$ determines a vector field on the infinite dimension manifold of functions $\tau$. We deduce, according to [33], that $\partial^{-1}\left(\Psi^{*}(t, \lambda) \Psi(t, \mu)\right)=\frac{1}{\tau(t)} X(t, \lambda, \mu) \tau(t)$.

Let $\Delta\left(s_{1}, \ldots, s_{n}\right)=\prod_{1 \leq j<i \leq n}\left(s_{i}^{-1}-s_{j}^{-1}\right)$, be the Vandermonde determinant. Fay identities (theorem 6.6) are generalized as follows. The $\tau$ function satisfies identities :

$$
\begin{aligned}
& \tau\left(t-\sum_{j=1}^{n}\left[y_{j}\right]\right) \Delta\left(y_{1}, \ldots, y_{n}\right)\left(\left(t-\sum_{j=1}^{n}\left[y_{j}\right]\right) \Delta\left(x_{1}, \ldots, x_{n}\right)\right)^{n-1} \\
& =\operatorname{det}\left(\left(t-\sum_{j=1}^{n}\left[x_{k}\right]+\left[x_{j}\right]-\left[y_{l}\right]\right) \Delta\left(x_{1}, \ldots, x_{j+1}, y_{j}, x_{j+1}, \ldots, x_{n}\right)\right)_{1 \leq j, l \leq n},
\end{aligned}
$$

and

$$
\left\{\psi \left(t, y_{1}^{-1}, \ldots, \psi\left(t, y_{n}^{-1}\right\}=e^{\sum_{j=1}^{\infty}} t_{j}\left(y_{1}^{-j}+\cdots+y_{n}^{-j}\right) \frac{\tau\left(t-\left[y_{l}\right]-\cdots-\left[y_{n}\right]\right.}{\tau(t)} \Delta\left(y_{1}, \ldots, y_{n}\right),\right.\right.
$$

where $\left\{u_{1}, \ldots, u_{n}\right\}$ is the Wronskian det $\left(\left(\frac{\partial}{\partial x}\right)^{j-1} u_{j}\right)_{1 \leq i, j \leq n}$.

We will see that $\tau$ functions characterize the KP hierarchy. Let $s_{j}(t)$ denote the elementary Schur polynomials, i.e., polynomials such that :

$$
e^{\xi(t, z)}=e^{\sum_{j=1}^{\infty} t_{j} z_{j}}=\sum_{j=1}^{\infty} s_{j}(t) z_{j}=1+t_{1} z+\left(\frac{1}{2} t_{1}^{2}+t_{2}\right) z^{2}+\left(\frac{1}{6} t_{1}^{3}+t_{1} t_{2}+t_{3}\right) z^{3}+\cdots
$$


with $s_{j}(t)=\frac{t_{1}^{j}}{j !}+\cdots+t_{n}$. By setting $\widetilde{\partial}=\left(\frac{\partial}{\partial t_{1}}, \frac{1}{2} \frac{\partial}{\partial t_{2}}, \frac{1}{3} \frac{\partial}{\partial t_{3}}, \ldots\right)$, we obtain

$$
\Psi(t, z)=\frac{\tau\left(t_{1}-z^{-1}, t_{2}-\frac{z^{-2}}{2}, t_{3}-\frac{z^{-3}}{3}, \ldots\right)}{\tau(t)} e^{\xi(t, z)}=\sum_{j=0}^{\infty} \frac{s_{j}(-\widetilde{\partial}) \tau(t)}{\tau(t)} \partial^{-j} e^{\xi(t, z)}=W(t) e^{\xi(t, z)},=
$$

where $W(t)=\sum_{j=0}^{\infty} \frac{s_{j}(-\widetilde{\partial}) \tau(t)}{\tau(t)} \partial^{-j}$, is the wave operator (6.7). Similarly, we have

$$
W^{-1}=\sum_{j=0}^{\infty} \partial^{-j} \frac{s_{j}(\widetilde{\partial}) \tau(t)}{\tau(t)}
$$

It follows from (6.8) that $L^{n}=W . \partial^{n} . W^{-1}$ and $L^{n}$ is expressed in terms of the $\tau$ function, $L^{n}=\sum_{i, j=0}^{\infty} \frac{s_{i}(-\widetilde{\partial}) \tau}{\tau} \partial^{n-i-j} \frac{s_{j}(\widetilde{\partial})}{\tau} \tau$. By developing this expression, we get

$$
L^{n}=\partial^{n}+n(\log \tau)^{\prime \prime} \partial^{n-2}+\cdots+\sum_{i+j=n+1} \frac{s_{i}(\widetilde{\partial}) \tau s_{j}(-\widetilde{\partial}) \tau}{\tau^{2}}+\cdots
$$

The formula (6.10) is written taking into account this last expression of $L^{n}$ and the relation (6.15) as follows :

$$
\frac{\partial}{\partial t_{n}}\left(1-\frac{\tau^{\prime}}{\tau} \partial^{-1}+\cdots\right)=\left(-\sum_{i+j=n+1} \frac{s_{i}(-\widetilde{\partial}) \tau s_{j}(-\widetilde{\partial}) \tau}{\tau^{2}} \partial^{-1}+\cdots\right)\left(1-\frac{\tau^{\prime}}{\tau} \partial^{-1}+\cdots\right) .
$$

Using the Hirota symbol ${ }^{1}$, we have

$$
\sum_{\substack{i+j=n+1 \\ i, j \geq 0}}\left(s_{i}(\widetilde{\partial}) \tau\right)\left(s_{j}(-\tilde{\partial}) \tau\right)=s_{n+1}(\widetilde{\partial}) \tau . \tau,
$$

and we obtain

$$
\tau^{2} \frac{\partial^{2}}{\partial t_{n} \partial t_{1}} \log \tau-\sum_{\substack{i+j=n+1 \\ i, j \geq 0}} s_{i}(\widetilde{\partial}) \tau s_{j}(-\widetilde{\partial}) \tau=0, \quad n \in \mathbb{N}^{*}
$$

These relations are called Hirota bilinear equations. They show that all the functions, $j \geq 2$, can be expressed in terms of the $\tau$ function. For example,

$$
u_{2}=\frac{\partial^{2}}{\partial t_{1}^{2}} \log \tau, \quad u_{3}=\frac{1}{2}\left(\frac{\partial^{3}}{\partial t_{1}^{3}}+\frac{\partial}{\partial t_{1}} \frac{\partial}{\partial t_{3}}\right) \log \tau, \quad u_{4}=\frac{1}{6}\left(\frac{\partial^{4}}{\partial t_{1}^{4}}-3 \frac{\partial^{2}}{\partial t_{1}^{2}} \frac{\partial}{\partial t_{2}}+2 \frac{\partial}{\partial t_{1}} \frac{\partial}{\partial t_{2}}\right) \log \tau-\left(\frac{\partial^{2}}{\partial t_{1}^{2}} \log \tau\right), \ldots
$$

In particular, these equations provide the KP equation in the following bilinear form :

$$
\frac{1}{12} \tau\left(\frac{\partial^{4} \tau}{\partial t_{1}^{4}}-4 \frac{\partial^{2} \tau}{\partial t_{1} \partial t_{3}}+3 \frac{\partial^{2} \tau}{\partial t_{2}^{2}}\right)-\frac{1}{3} \frac{\partial \tau}{\partial t_{1}}\left(\frac{\partial^{3} \tau}{\partial t_{1}^{3}}-\frac{\partial \tau}{\partial t_{3}}\right)+\frac{1}{4}\left(\frac{\partial^{2} \tau}{\partial t_{1}^{2}}+\frac{\partial \tau}{\partial t_{2}}\right)\left(\frac{\partial^{2} \tau}{\partial t_{1}^{2}}-\frac{\partial \tau}{\partial t_{2}}\right)=0
$$

Therefore, we have

Theorem 6.7. The $\tau$ functions characterize the KP hierarchy.

The equations of soliton theory play an important role in the characterization of Jacobian varieties. Let

$$
\mathscr{H}_{g}=\left\{Z \in M_{g}(\mathbb{C}): \Omega=\Omega^{\top}, I \Omega>0\right\}
$$

\footnotetext{
$\left.{ }^{1} p\left(\partial_{t}\right) f(t) \cdot g(t) \equiv p\left(\frac{\partial}{\partial s_{1}}, \frac{\partial}{\partial s_{2}}, \ldots\right) f(t+s) g(t-s)\right|_{s=0}$ where $p$ is any polynomial, $f(t)$ and $g(t)$ are two differentiable functions.
} 
be the Siegel half-space, $\Lambda=\mathbb{Z}^{g} \oplus Z \mathbb{Z}^{g}$ a lattice in $\mathbb{C}^{g}$ and $T=\mathbb{C}^{g} / \Lambda$ a principally polarised Abelian variety. We show $[24,37]$ that the following three conditions are equivalent : (i) There are vector fields $v_{1}, v_{2}, v_{3}$ on $\mathbb{C}^{g}$ and a quadratic form $q(t)=\sum_{k, l=1}^{3} q_{k l}(t) t_{k} t_{l}$, such that : for all $z \in \mathbb{C}^{g}$, the function $\tau(t)=e^{q(t)} \theta\left(\sum_{k=1}^{3} t_{k} v_{k}+z\right)$, satisfies the KP equation. The theta divisor does not contain an Abelian subvariety of $T$ for which the vector $v_{1}$ is tangent. (ii) $T$ is isomorphic to the Jacobian variety of a reduced non-singular complete curve of genus $g$. (iii) There is a matrix $\mathscr{V}=\left(v_{1}, v_{2}, \ldots\right)$ of order $g \times \infty, v_{k} \in \mathbb{C}^{g}$, of rank $g$ and a quadratic form $Q(t)=\sum_{k, l=1}^{\infty} q_{k l}(t) t_{k} t_{l}$, such that: for all $z \in \mathbb{C}^{g}, \widetilde{\tau}(t)=e^{Q(t)} \theta(\mathscr{V} t+z)$, is a $\tau$ function for KP hierarchy.

\section{References}

[1] D. J. Korteweg, G. de Vries, On the change of form of long waves advancing in a rectangular canal and on a new type of long stationary waves, Phil. Mag., 39 (1895), 422-443.

[2] J. Boussinesq, Essai sur la théorie des eaux courantes, Mémoires présentés par divers savants, Acad. des Sci. Inst. Nat. France, XXIII (1877), 1-680.

[3] J. Scott-Russell, Report on Waves. Report to the 14th meeting British Association for Advancement of Science, (1844), 311-390.

[4] I. M. Gel'fand, B. M. Levitan, On the determination of a differential equation from its spectral function, Amer. Math. Soc. Transl., 2 (1) (1955), 253-304.

[5] N. Levinson, The inverse Sturm-Liouville problem, Mat. Tidsskr. B., (1949) 25-30.

[6] M. D. Kruskal, R. M. Miura, C. S. Gardner, N. J. Zabusky, Korteweg-de Vries equation and generalizations V. Uniqueness and nonexistence of polynomial consevation laws, J. Math. Phys., 11 (1970), 952-960.

[7] P. Lax, Integrals of nonlinear equations of evolution and solitary waves, Comm. Pure Appl. Math., 21 (1968), 467-490.

[8] C. S. Gardner, J. M. Greene, M. D. Kruskal, R. M. Miura, Method for solving the Korteweg-de Vries equation, Phys. Rev. Lett., 19 (1967), 1095-1097.

[9] C. S. Gardner, Korteweg-de Vries equation and generalizations IV, The Korteweg-de Vries equation as a Hamiltonian system, J. Math. Phys., 12 (1971), 1548-1551.

[10] C. S. Gardner, J. M. Greene, M. D. Kruskal, R. M. Miura, Korteweg-de Vries equation and generalizations VI, Methods for exact solutions, Comm. Pure Appl. Math., 27 (1974), 97-133.

[11] R. M. Miura, C. S. Gardner, M. D. Kruskal, Korteweg-de Vries equation and generalizations II. Existence of conservation laws and constants of motion, J. Math. Phys., 9 (1968), 1204-1209.

[12] P. Lax, Periodic solutions of the KdV equation, Comm. Pure Appl. Math., 28 (1975), 141-188.

[13] P. Lax, R. Phillips, Scattering theory for automorphic functions. Annals Math. Studies, Princeton, 1976.

[14] A. Lesfari, Etude des équations stationnaire de Schrödinger, intégrale de Gelfand-Levitan et de Korteweg-de-Vries. Solitons et méthode de la diffusion inverse, Aequat. Math., 85 (2013), 243-272.

[15] M. Wadati, M. Toda, The exact N-soliton solution of the Korteweg-de Vries equation, J. Phys. Soc. Japan, 32 (1972), 1403-1411.

[16] B. B. Kadomtsev, V I. and Petviashvili, On the Stability of Solitary Waves in Weakly Dispersing Media, Sov. Phys. Dokl., 15 (6) (1970), 539-541.

[17] M. Sato, Soliton equations and the universal Grassmann manifold, Math. Lect. Note Ser. 18. Sophia University, Tokyo, 1984.

[18] P. van Moerbeke, Integrable foundations of string theory in Lectures on Integrable Systems, (Sophia-Antipolis, 1991), World Sci., River Edge, N.J., (1994), 163-267.

[19] J.-L. Gervais, Infinite family of polynomial functions of the Virasoro generators with vanishing Poisson bracket, Phys. Lett. B, 160 (4-5) (1985), 277-278.

[20] M. Adler, P. van Moerbeke, P. Vanhaecke, Algebraic integrability, Painlevé geometry and Lie algebras, A series of modern surveys in mathematics, Volume 47, Springer-Verlag, 2004. 
[21] A. Lesfari, Abelian surfaces and Kowalewski's top, Ann. Scient. École Norm. Sup., Paris, $4^{e}$ série, .21 (1988), 193-223.

${ }^{[22]} \mathrm{M}$. Adler, On a trace functional for formal pseudo differential operators and the symplectic structure of the Korteweg-de Vries type equations, Invent. Math., 50(3) (1979), 219-248.

[23] B. Kostant, The solution to a generalized Toda lattice and representation theory, Adv. Math., 34 (3) (1979), 195-338.

[24] W. Symes, Systems of Toda type, inverse spectral problems and representation theory, Invent. Math., 59 (1980), 13-53.

[25] A. I. Belokolos, V. Z. Bobenko, V.Z., Enol'skii, A. R. Its, V. B. Matveev, Algebro-Geometric approach to nonlinear integrable equations, Springer-Verlag, 1994.

[26] A. Lesfari, Integrables Hamiltonian systems and the isospectral deformation method, Int. J. of Appl. Math. And Mech., 3 (4) (2007), 35-55.

[27] A. Lesfari, Théorie spectrale et problèmes non-linéaires, Surv. Math. Appl., 5 (2010), 151-190.

[28] A. Lesfari, Algèbres de Lie affines et opérateurs pseudo-différentiels d'ordre infini, Math. Rep., 14 (64),1 (2012), 43-69 (2012).

[29] A. Lesfari, Introduction à la géométrie algébrique complexe, Éditions Hermann, Paris, 2015.

[30] I. M. Gel'fand, L. Dickey, Family of Hamiltonian structures connected with integrable nonlinear differential equations, Funct. Anal. Appl., 2 (1968), 92-93.

[31] L. Dickey, Soliton equations and integrable systems, World Scientific, 1991.

${ }^{[32]}$ L. Dickey, Additional symmetries of KP, Grassmannian and the string equation, Mod. Phys. Lett., A 8 (1993), 1259-1272.

[33] L. Dickey, Lectures on classical W-algebras, Acta Appl. Math., 47 (1997), 243-321.

[34] E. Date, M. Jimbo, M. Kashiwara, T. Miwa, Transformation groups for soliton equations, Proc. RIMS Symp. Nonlinear integrable systems (Kyoto, 1981). Classical and quantum theory, Singapore, World Scientific (1983), 39-119.

[35] M. Sato, The KP hierarchy and infinite-dimensional Grassmann manifolds, Proc. of Sympos. Pure Math., 49 (1989), 51-66.

${ }^{[36]}$ M. Sato, Y. Sato, Soliton equations as dynamical systems on infinite dimensional Grassmann manifolds, Lect. Notes in Num. Appl. Anal., 5 (1982), 259-271.

[37] T. Shiota, Characterization of jacobian varieties in terms of soliton equations, Invent. Math., 83 (1986), 333-382. 